\title{
Toward a Dynamic Logic of Questions
}

\author{
Johan van Benthem • Ştefan Minică
}

Received: 1 August 2010 / Accepted: 31 August 2011 / Published online: 10 June 2012 (C) The Author(s) 2012. This article is published with open access at Springerlink.com

\begin{abstract}
Questions are triggers for explicit events of 'issue management'. We give a complete logic in dynamic-epistemic style for events of raising, refining, and resolving an issue, all in the presence of information flow through observation or communication. We explore extensions of the framework to multiagent scenarios and long-term temporal protocols. We sketch a comparison with some alternative accounts.
\end{abstract}

Keywords Question • Issue management • Logical dynamics

\section{Introduction and Motivation}

Questions are different from statements, but they are just as important in driving reasoning, communication, and general processes of investigation. The first logical studies merging questions and propositions seem to have come from the Polish tradition: cf. [40]. A forceful modern defender of this dual perspective is Hintikka, who has long pointed out how any form of inquiry depends on an interplay of inference and answers to questions. Cf. [15] and

\footnotetext{
J. van Benthem $(\varangle) \cdot$ Ş. Minică

Institute for Logic, Language \& Computation,

University of Amsterdam, P.O. Box 94242,

1090 GE Amsterdam, The Netherlands

e-mail: johan@science.uva.nl

Ş. Minică

e-mail: stefan.minica@gmail.com

J. van Benthem

Department of Philosophy, Stanford University,

Stanford, California 94305, USA
} 
[14] on the resulting 'interrogative logic', and the epistemological views behind it. These logics are mainly about general inquiry and learning about the world. But there is also a related stream of work on questions in natural language, as important speech acts with a systematic linguistic vocabulary. Key names are Groenendijk \& Stokhof: cf. [10,12], and the recent 'inquisitive semantics' of [11] ties this in with a broader information-oriented 'dynamic semantics'. Logic of inquiry and logic of questions are related, but there are also differences in thrust: a dynamic logic of 'issue management' that fits our intuitions is not necessarily the same as a logic of speech acts that focuses on what natural language provides.

In this paper, we do not choose between these streams, but we propose a different technical approach. Our starting point is a simple observation. Questions are evidently important informational actions in human agency. Now the latter area is the birth place of dynamic-epistemic logic of explicit events that make information flow. But surprisingly, existing dynamic-epistemic systems do not give an explicit account of what questions do. In fact, central examples in the area have questions directing the information flow (say, by the Father in the puzzle of the Muddy Children) - but the usual representations in systems like $P A L$ or $D E L$ leave them out, and merely treat the answers, as events of public announcement. Can we make questions themselves first-class citizens in dynamic-epistemic logic, and get closer to the dynamics of inquiry? In [4], Baltag has shown that we can. We will take this further, following a methodology that has already worked in other areas, and pursuing the same issues here: what are natural acts of inquiry, and how can dynamic logics bring out their structure via suitable recursion axioms? Moreover, by doing so, we at once get an account of non-factual questions, multi-agent scenarios, syntactic aspects, temporal sequences, and other themes that have already been studied in a $D E L$ setting.

Overview Our analysis starts with Section 2 on a static logic of information and public questions, a natural expansion of epistemic logic. Section 3 then identifies key dynamic actions of 'issue management', discusses some of their properties, and presents a complete dynamic logic. We follow up with two themes showing how this system leads to natural extensions: multiagent perspectives on public and private questions in Section 4, and temporal protocols that regulate what questions can be asked (and answers can be given) in Section 5. In Section 6, we discuss where we stand with this 'proof of concept', and which further directions look promising. Section 7 is a very brief comparison with existing 'logic of interrogation' and 'inquisitive logic'.

\section{A Toy System of Information and Issues}

The methodology of dynamic-epistemic logic starts with a static base logic describing states of some informational phenomenon, and identifies relevant informational state-changing events. Then, dynamic modalities are added to 
the base language, and their complete logic is determined on top of the given logic of the static models. To work in the same style, we first need a convenient static semantics to 'dynamify'. We take such a model from existing semantics of public questions, considering only one agent first, for simplicity. We will work in the style of epistemic logic and public announcement logic $P A L$, though our dynamic logic of questions will also have its differences.

\subsection{Epistemic Issue Models}

We work over standard epistemic models. In this setting, a simple framework for representing questions uses an equivalence relation over some relevant domain of alternatives, that we will call the 'issue relation'. This idea is found in many places, from linguistics (cf. [12]) to learning theory (cf. [19]): the current 'issue' is a partition of the set of options, with partition cells standing for the areas where we would like to be. This partition may be induced by a conversation whose current focus are the issues that have been put on the table, or a game where finding out about certain issues has become important to further play, a learning scenario for the language fed to us by our environment, or even a whole research program with an agenda determining what is currently under investigation. The 'alternatives' or worlds may range here from simple finite settings like deals in a card game to complex infinite histories representing a total life experience. Formally, all this reduces to:

Definition 1 (Epistemic Issue Model) An epistemic issue model is a structure $M=\langle W, \sim, \approx, V\rangle$ where:

- $\quad W$ is a set of possible worlds or states (epistemic alternatives),

- $\sim$ is an equivalence relation on $W$ (epistemic indistinguishability),

- $\approx$ is an equivalence relation on $W$ (the abstract issue relation),

- $\quad V: \mathrm{P} \rightarrow \wp(W)$ is a valuation for atomic propositions $p \in \mathrm{P}$.

We could introduce models with more general relations, to account for, say, lack of epistemic introspection into the current issue, or belief instead of knowledge. While this is important eventually, equivalence relations will suffice for the points that we will make in this paper.

In Fig. 1 we illustrate the previous formal definition graphically. Here and in subsequent diagrams, epistemic indistinguishability is represented by lines linking possible worlds, and the issue relation is represented by partition cells. We use the usual conventions and skip reflexive and transitive relations. We assume that the actual world is the top left one and in some cases, we will use double lines instead of partition cells to represent issue relations. With this understanding, Fig. 1 depicts, from left to right, an epistemic issue model in which nothing is known and everything is an issue, a second one in which $q$ is known in the actual world and the issue is to find out about $p$, and, finally, one in which everything is known in the actual world, and nothing is an issue. 

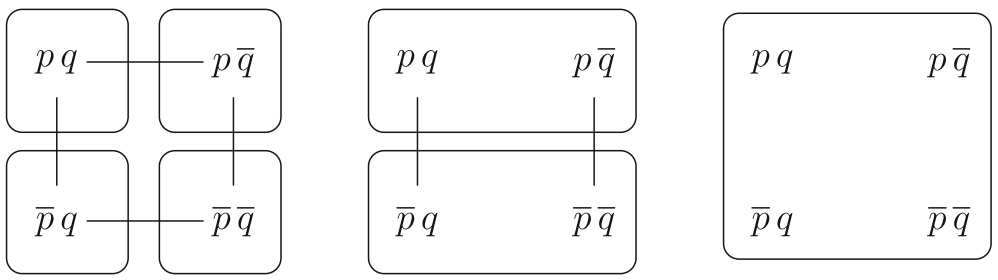

Fig. 1 Examples of epistemic issue models

\subsection{Information and Issues: Language and Semantics}

To work with these structures, we need matching modalities in our language. Here we make a minimal choice of modal and epistemic logic for state spaces plus two modalities describing the issue structure. First, $K \varphi$ talks about knowledge or semantic information of an agent, its informal reading is ' $\varphi$ is known', and its explanation is as usual: ' $\varphi$ holds in all epistemically indistinguishable worlds'. To describe our models a bit further, we add a universal modality $U \varphi$ saying that ' $\varphi$ is true in all worlds'. Next, we use $Q \varphi$ to say that, locally in a given world, the current structure of the issue-relation has $\varphi$ true: ' $\varphi$ holds in all issue-equivalent worlds'. While convenient, this local notion does not express the global assertion that the current issue is $\varphi$, which will be defined later.

Finally, we find a need for a notion that mixes the epistemic and issue relations, talking (roughly) about what would be the case if the issue were resolved given what we already know. Technically, we add an intersection modality $R \varphi$ saying that " $\varphi$ holds in all epistemically indistinguishable and issue equivalent worlds". While such modalities are frequent in many settings, they complicate axiomatization. We will assume the standard device of adding nominals naming single worlds (cf. $[9,20]$ for recent instances of this technique in the $D E L$ setting). ${ }^{1}$

Definition 2 (Static Language) The language $\mathcal{L}_{\mathbf{E L}_{\mathbf{Q}}}(\mathrm{P}, \mathrm{N})$ has disjoint countable sets $\mathrm{P}$ and $\mathrm{N}$ of propositions and nominals, respectively, with $p \in \mathrm{P}, i \in \mathrm{N}$. Its formulas are defined by the following inductive syntax rule:

$$
i|p| \perp|\neg \varphi|(\varphi \wedge \psi)|U \varphi| K \varphi|Q \varphi| R \varphi
$$

When needed, dual existential modalities $\widehat{U}, \widehat{K}, \widehat{Q}$ and $\widehat{R}$ are defined as usual. Shortcuts to express disjunction and other boolean connectives are also used

\footnotetext{
${ }^{1}$ As one illustration, working with nominals requires a modified valuation function in Definition 1, to a $V: \mathrm{P} \uplus \mathrm{N} \rightarrow \wp(W)$ mapping every proposition $p \in \mathrm{P}$ to a set of states $V(p) \subseteq W$, but every nominal $i \in \mathrm{N}$ to a singleton set $V(i)=w$ of a world $w \in W$.
} 
in their standard way. Formulas in this static language receive their meaning in the following way:

Definition 3 (Interpretation) Formulas are interpreted in models $M$ at worlds $w$ by the following recursive clauses:

$$
\begin{array}{lll}
M \models_{w} p & \text { iff } & w \in V(p), \quad M \models_{w} i \text { iff } w \in V(i), \\
M \models_{w} \neg \varphi & \text { iff } & \text { not } M \models_{w} \varphi, \quad M \models_{w} \varphi \wedge \psi \text { iff } M \models_{w} \varphi \text { and } M \models_{w} \psi, \\
M \models_{w} U \varphi & \text { iff } & \text { for all } w \in W: M \models_{w} \varphi, \\
M \models_{w} K \varphi & \text { iff } & \text { for all } v \in W: w \sim v \text { implies } M \models_{v} \varphi, \\
M \models_{w} Q \varphi & \text { iff } & \text { for all } v \in W: w \approx v \text { implies } M \models_{v} \varphi, \\
M \models_{w} R \varphi & \text { iff } & \text { for all } v \in W: w(\sim \cap \approx) v \text { implies } M \models_{v} \varphi .
\end{array}
$$

For instance, with this language we can express that the structure of the current issue settles fact $\varphi$ with the following formula:

$$
U(Q \varphi \vee Q \neg \varphi)^{2}
$$

Here is how we say that an agent considers it possible that fact $\varphi$ is not settled by the structure of the current issue:

$$
\widehat{K}(\varphi \wedge \widehat{Q} \neg \varphi)
$$

The next example says that an agent knows locally that a certain fact $\varphi$ would be settled by the issue, while it is not settled globally:

$$
K Q \varphi \wedge \neg U(Q \varphi \vee Q \neg \varphi)
$$

As for the third modality of 'resolution', it describes intuitively what agents would know if the current issue is resolved. Thus, we can say that in the current epistemic situation $\varphi$ is neither known by the agent nor settled by the structure of the issue, but it is true upon resolution:

$$
\neg Q \varphi \wedge \neg K \varphi \wedge R \varphi
$$

A more complex example is when a fact is neither known nor settled in any world of the model, but it is true in all indistinguishable and issue-equivalent worlds, and it would be settled by a resolution action:

$$
\neg \widehat{U}(K \varphi \vee Q \varphi) \wedge U R \varphi
$$

These examples show that our language can express quite complex notions about questions. Many such notions have been considered in the literature

\footnotetext{
${ }^{2}$ We use the term 'settling' in a technical sense, as saying that the issue answers (either explicitly or implicitly) the question whether $\varphi$ holds. In natural language, there is also the notion of 'settling an issue', an event of finding out which partition cell we are in. This will be one of our later actions of 'issue management', that of resolution.
} 
about questions and information flow, but often restricted to factual questions, and without the benefit of a uniform formal language.

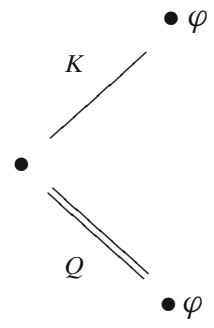

We end with a technical point: the intersection modality $R \varphi$ cannot be defined in terms of $K$ and $Q$. In particular, $\widehat{R} \varphi$ is not equivalent with $\widehat{K} \varphi \wedge \widehat{Q} \varphi$, witness the counterexample above. However, the use of 'nominals' $i$ from hybrid logic helps us to completeness, by the valid converse:

$$
\widehat{K}(i \wedge \varphi) \wedge \widehat{Q}(i \wedge \varphi) \rightarrow \widehat{R} \varphi
$$

\subsection{Static Logic of Information and Issues}

As for reasoning with our language, we have the valid implications $\widehat{K} \varphi \rightarrow \widehat{U} \varphi$, $\widehat{Q} \varphi \rightarrow \widehat{U} \varphi, \widehat{R} \varphi \rightarrow \widehat{U} \varphi$. The following are not, in general, valid implications $R \varphi \rightarrow Q \varphi, R \varphi \rightarrow \neg Q \varphi, K \varphi \rightarrow Q \varphi, Q \varphi \rightarrow K \varphi, R \varphi \rightarrow K \varphi$.

More generally, we write $\models \varphi$ if the static formula $\varphi$ is true in every model at every world. The static epistemic logic $\mathbf{E} \mathbf{L}_{\mathbf{Q}}$ of information and questions in our models is the set of all validities:

$$
\mathbf{E L}_{\mathbf{Q}}=\left\{\varphi \in \mathcal{L}_{\mathbf{E} \mathbf{L}_{\mathbf{Q}}}: \models \varphi\right\}
$$

Definition 4 (Axiomatization) The proof system $E L_{Q}$ contains the customary (epistemic) S5 axioms for K, Q and R:

1. $K p \rightarrow p$ (Truth), $K p \rightarrow K K p, \neg K p \rightarrow K \neg K p$ (Full Introspection)

2. $p \rightarrow Q \widehat{Q} p, p \rightarrow \widehat{Q} p, \widehat{Q} \widehat{Q} p \rightarrow \widehat{Q} p$ (equivalence relation for issues),

3. $p \rightarrow R \widehat{R} p, p \rightarrow \widehat{R} p, \widehat{R} \widehat{R} p \rightarrow \widehat{R} p$ (equivalence relation for resolution),

together with the characteristic axiom for intersection:

4. $\widehat{K} i \wedge \widehat{Q} i \leftrightarrow \widehat{R} i$.

In addition, it contains a standard hybrid logic with a universal modality:

5. $\square(p \rightarrow q) \rightarrow(\square p \rightarrow \square q), \square \in\{U, K, R, Q\}$ (Distribution)

6. $\neg \square \neg p \leftrightarrow \diamond p, \diamond, \square \in\{U, K, R, Q\}$ (Duality)

7. $p \rightarrow U \widehat{U} p, p \rightarrow \widehat{U} p, \widehat{U} \widehat{U} p \rightarrow \widehat{U} p, \widehat{U} i, \diamond p \rightarrow \widehat{U} p, \diamond \in\{\widehat{K}, \widehat{R}, \widehat{Q}\}$,

8. $\diamond(i \wedge p) \rightarrow \square(i \rightarrow p), \square \in\{U, K, R, Q\}$ (Nominals) 
9. From $\vdash_{\mathrm{PC}} \varphi$ infer $\varphi$ (Prop), From $\varphi$ and $\varphi \rightarrow \psi$ infer $\psi$ (MP)

10. From $\varphi$ infer $\square \varphi$, for $\square \in\{U, K, R, Q\}$ (Necessitation)

11. From $\varphi$ and $\sigma_{\text {sort }}(\varphi)=\psi$ infer $\psi$, where $\sigma_{\text {sort }}$ is 'sorted' ${ }^{3}$

12. From $i \rightarrow \varphi$ infer $\varphi$, for $i$ not occurring in $\varphi$

13. From $\widehat{U}(i \wedge \diamond j) \rightarrow \widehat{U}(j \wedge \varphi)$ infer $\widehat{U}(i \wedge \square \varphi)$, for $\diamond \in\{\widehat{K}, \widehat{R}, \widehat{Q}\}, i \neq j$, and $j$ not occurring in $\varphi$.

We write $\vdash_{E L_{Q}} \varphi$ if $\varphi$ is provable in the proof system $E L_{Q}$. These laws of reasoning derive many intuitive principles. For instance, here is the simple proof that agents have introspection about the current public issue:

$$
U(Q p \vee Q \neg p) \quad \vdash_{E L_{Q}} \quad U U(Q p \vee Q \neg p) \quad \vdash_{E L_{Q}} \quad K U(Q p \vee Q \neg p)
$$

Here are some simple derivable principles connecting our modalities:

$$
U \varphi \rightarrow K \varphi, \quad U \varphi \rightarrow Q \varphi, \quad U \varphi \rightarrow R \varphi \quad K \varphi \rightarrow R \varphi, \quad Q \varphi \rightarrow R \varphi
$$

Further technical details of proofs are irrelevant to our purposes here. We refer to [23] for hybrid modal proof systems and completeness theorems. This standard machinery leads to the expected result:

Theorem 1 (Completeness of $\mathbf{E L}_{\mathbf{Q}}$ ) For every formula $\varphi \in \mathcal{L}_{\mathbf{E L}_{\mathbf{Q}}}(\mathrm{P}, \mathrm{N})$ :

$$
\models \varphi \quad \text { if and only if } \vdash_{E L_{Q}} \varphi
$$

\section{Dynamic Logic of Issue Management}

In dynamic epistemic logic, the next step is now to identify basic events of information flow, and expand the logic accordingly. This situation is very analogous with logic of questions and events of 'issue management'.

\subsection{Basic Actions of Issue Management}

To identify basic actions that change the issue relation in a given model, we first look at some pictures. For simplicity, we start with the initial issue as the universal relation, represented as a frame border.

In Fig. 2, the first transition records the effect of asking a question: the issue relation is split into $p$ and $\neg p$ cells. The second transition illustrates the effect of asking a second question: the issue partition is further refined.

In Fig. 3, the first transition is an announcement: the indistinguishability links between $p$ and $\neg p$ worlds are removed. The second transition shows how a second announcement further refines the epistemic partition. Here and

\footnotetext{
${ }^{3}$ The technical notion 'sorted' and its uses are explained in [23].
} 


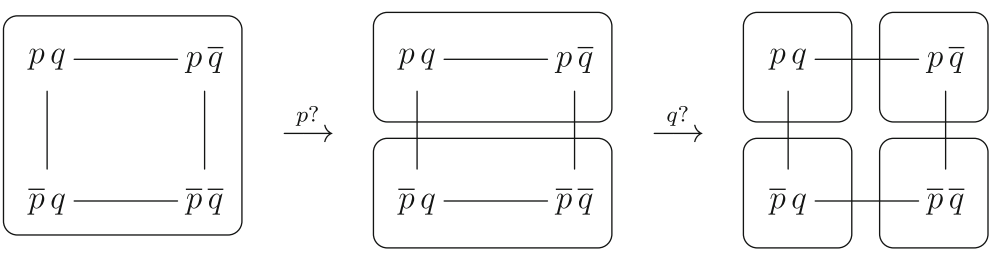

Fig. 2 Effects of asking yes/no questions

henceforth, we use a special sort of event that is congenial to this setting, viz. the link-cutting announcements $\varphi$ ! of van Benthem and Liu [31]. These do not throw away worlds, but merely cut all links between $\varphi$ - and $\neg \varphi$-worlds, keeping the whole model available for further reference.

In this way, there is a symmetry between a question and a soft announcement. One refines the issue, the other the information partition:

Definition 5 (Questions \& Announcements) Let $\stackrel{\varphi}{\underline{\underline{\varphi}}}_{M}=\left\{(w, v) \mid\|\varphi\|_{w}^{M}=\right.$ $\left.\|\varphi\|_{v}^{M}\right\}$. Executing action $\varphi$ ? in $M$ results in $M_{\varphi ?}=\left\langle W_{\varphi ?}, \sim_{\varphi ?}, \approx_{\varphi ?}, V_{\varphi ?}\right\rangle$, while a $\varphi$ ! action results in $M_{\varphi !}=\left\langle W_{\varphi !}, \sim_{\varphi !}, \approx_{\varphi !}, V_{\varphi !}\right\rangle$, where we put:

$$
\begin{array}{ll}
W_{\varphi ?}=W & W_{\varphi !}=W \\
\sim_{\varphi ?}=\sim & \sim_{\varphi !}=\sim \cap \underline{\underline{\underline{\varphi}}}_{M} \\
\approx_{\varphi ?}=\approx \cap \underline{\underline{\underline{\varphi}}}_{M} & \approx_{\varphi !}=\approx \\
V_{\varphi ?}=V & V_{\varphi !}=V
\end{array}
$$

The symmetry in this mechanism would be lost if we let $p$ ! be an executable action only if it is truthful. For, the corresponding question $p$ ? is executable in every world in a model, even those not satisfying $p$. The results that will follow can easily be stated for both kinds of announcement: truthful or not.

One attractive feature of this setting is that it suggests further natural operations on information and issues. In particular, Fig. 4 contains two more management actions. In the first example two Yes/No questions $p$ ? and $q$ ? are asked, and then a global resolving action follows on the epistemic relation. In the second, two announcements $p$ ! and $q$ ! are made, and a refinement action

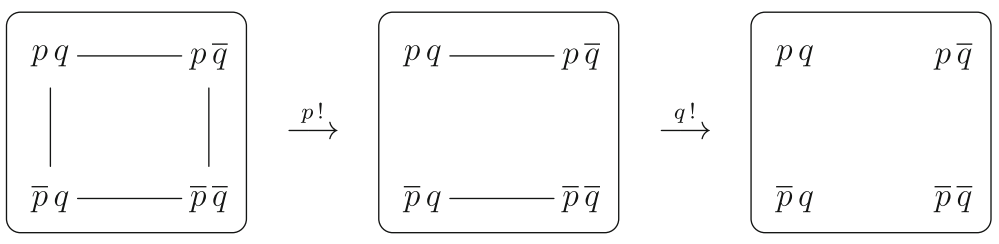

Fig. 3 Almost symmetrical effects of 'Soft' announcing 


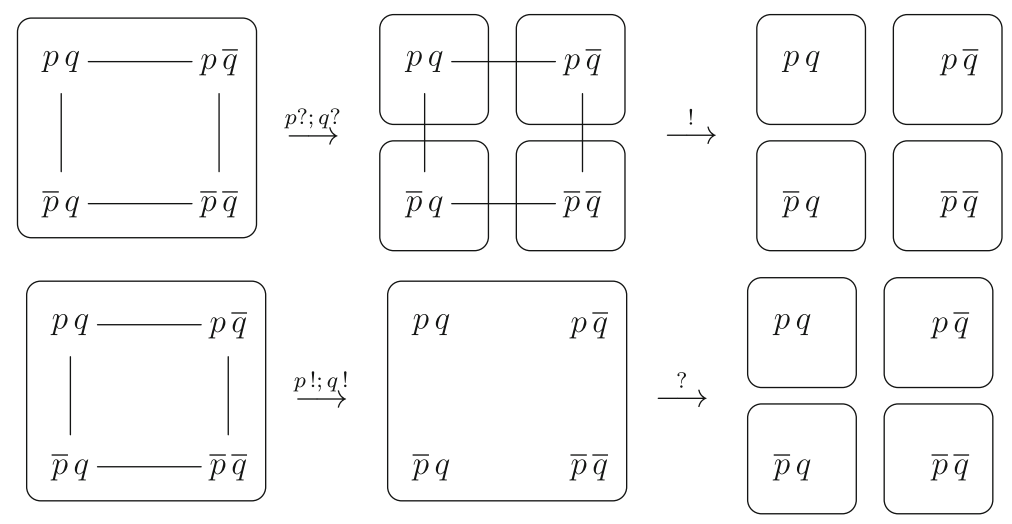

Fig. 4 Resolving and refining actions

follows on the issue relation, adjusting it to what agents already know. These operations are natural generalizations of asking and announcing:

Definition 6 (Resolution and Refinement) An execution of the 'resolve' action !, and of the 'refine' action ? in model $M$ results in changed models $M_{!}=\left\langle W_{!}, \sim_{!}, \approx_{!}, V_{!}\right\rangle, M_{?}=\left\langle W_{?}, \sim_{?}, \approx_{?}, V_{?}\right\rangle$, respectively, with:

$$
\begin{array}{ll}
W_{?}=W & W_{!}=W \\
\sim_{?}=\sim & \sim_{!}=\sim \cap \approx \\
\approx_{?}=\approx \cap \sim & \approx_{!}=\approx \\
V_{?}=V & V_{!}=V
\end{array}
$$

Again, the two actions are symmetric. As a way of understanding this, we could introduce a new agent whose role is that of an 'issue manager', dual to the epistemic information agent.

It is also useful to have one more issue management action \# that simultaneously changes both equivalence relations. ${ }^{4}$ The effect of executing this in model $M$ is a new model $M_{\#}=\left\langle W_{\#}, \sim_{\#}, \approx_{\#}, V_{\#}\right\rangle$ with:

$$
W_{\#}=W, \quad \sim_{\#}=\approx_{\#}=\sim \cap \approx, \quad V_{\#}=V
$$

Here is a summary of our repertoire of issue management actions:

$$
\begin{array}{cccc}
{[\varphi !]} & \text { 'Soft' announcement } & {[\varphi ?]} & \text { Question } \\
{[!]} & \text { Resolution } & {[?]} & \text { Refinement } \\
{[\#]} & \text { Simultaneous resolution } & \text { or } & \text { 'parallel refinement' }
\end{array}
$$

\footnotetext{
${ }^{4}$ Eric Pacuit (p.c.) has suggested that there may also be natural actions of simplifying a current issue, which would result in coarsening the current partition.
} 


\subsection{Semantic Properties of Issue Management}

Our basic actions satisfy some intuitive principles. In particular, our three last ones form an algebra under composition, witness the following table:

\begin{tabular}{c|ccc}
$;$ & $!$ & $?$ & $\#$ \\
\hline$!$ & $!$ & $\#$ & $\#$ \\
$?$ & $\#$ & $?$ & $\#$ \\
$\#$ & $\#$ & $\#$ & $\#$
\end{tabular}

With more specific management actions of questions and announcements, the picture is more diverse. In particular, composing these operations is complex, and many prima facie laws do not hold, for instance:

Fact 1 (Composition) The following equations are not valid in $D E L_{Q}$ :
(11) $\varphi$ !; ! = !; $\varphi$ !
(12) $\varphi$ !; ? = ?; $\varphi$ !
(13) $\varphi$ !; \# =\#; $\varphi$ !
(14) $\varphi$ ?; ! = !; $\varphi$ !
(15) $\varphi$ ?; ? = ?; $\varphi$ !
(16) $\varphi$ ?; \# =\#; $\varphi$ ?
(17) $\varphi$ ?; $\psi !=\psi ! ; \varphi$ ?

Proof The following table lists simple counter-examples, with the following understanding. Each line presents a three-world static starting model $i, j, k$ with information cells between round brackets $($, ) and issue cells between square brackets [, ]. The relevant formulas use nominals for the worlds.

\begin{tabular}{|cccc|}
\hline No. & EIM & $\varphi$ & $\psi$ \\
11 & $([i j][k])$ & $\widehat{K} k \wedge \widehat{Q} j \wedge \neg i$ & \\
12 & {$[(i j)(k)]$} & $\widehat{Q} k \wedge \widehat{K} j \wedge \neg i$ & \\
13 & $([i j])([k])$ & $i$ & \\
14 & $([i j k])$ & $i$ & \\
15 & {$[(i j k)]$} & $j$ & \\
16 & $([i j])([k])$ & $i$ & \\
17 & $([i j k])$ & $i$ & $\widehat{R} i$ \\
\hline
\end{tabular}

Some of these examples crucially involve non-factual formulas. For instance, $\varphi$ ?; $\psi !=\psi ! ; \varphi$ ? and $\varphi$ ?; $\psi$ ? = $\varphi$ ? $\cdot \psi$ ? only fail for formulas $\varphi$ with non-factual content. They are both valid for factual $\varphi$. This is so because $V_{M}=V_{\varphi}$ ? $=V_{\varphi !}$ : the valuation function remains unchanged after questioning actions. Then, by a simple inductive argument, for old models $M$ and updated $M^{\prime}$, we have $\llbracket \psi \rrbracket_{M}=\llbracket \psi \rrbracket_{M^{\prime}}$ for all purely propositional formulas $\psi$ : essentially, their extensions in epistemic-issue models are independent of epistemic or issue structure.

Next, let us see how some known features of information flow in public announcement logic $P A L$ fare with our issue management actions. 
Repetition In $P A L$, repeating the same assertion ! $\varphi$ has no new effects when its content $\varphi$ is factual. But as the Muddy Children puzzle shows, repeating the same epistemic assertion can be informative, and lead to new effects, or in the above short-hand notation: $\varphi ! ; \varphi ! \neq \varphi !$ The reason is that when the model has changed, epistemic operators may change truth values. What about $D E L_{Q}$ : is asking a question once the same as asking it twice? Again, for factual questions, this is clearly so, given the above semantics: the issue relation no longer changes in the second step. But when the question itself can refer to the issue relation, things are different:

Fact 2 (Iteration) The equation $\varphi$ ?; $\varphi$ ? $=\varphi$ ? is invalid in $D E L_{Q}$.

Proof Take $\xi:=(\widehat{Q} i \rightarrow(j \vee k)) \wedge\left(\left(\widehat{Q}_{j} \wedge p\right) \rightarrow \widehat{Q} i\right)$. Two successive updates with this question, computed as above, each change a model with three worlds $i, j, k$, a universal issue relation, and $p$ true at $k$.

Composition Next comes a difference with $P A L$. Public announcement satisfies the valid composition principle: $\varphi ! ; \psi !=(\varphi \wedge[\varphi] \psi)$ ! It was observed in [31] and [30], that this does not hold for more complex model changes. ${ }^{5}$

Fact 3 (Proper Iteration) There is no question composition principle.

Proof If there were one single assertion having just the same effect as a sequence $\varphi$ ?; $\psi$ ?, then, starting with the issue configured as the universal relation on the domain of a model, such a sequence will always induce a two, not four, element partition; this refutation is also depicted in Fig. $4 .^{6}$

Related to this are dynamic properties of ordering. While action order makes no difference with purely factual assertions or questions, it does when the content may be of an explicit epistemic or issue-related nature.

We have seen that information update and questions have many subtleties. It is time for a dynamic epistemic logic of issues that can reason about these.

\subsection{Issue Management: Language and Semantics}

In order to talk explicitly about the above changes, dynamic modalities are added to the earlier static language of information and issues:

Definition 7 (Dynamic Language) Language $\mathcal{L}_{\mathbf{D E L}_{\mathbf{Q}}}(\mathrm{P}, \mathrm{N})$ is defined by adding the following clauses to Definition 2: $[\varphi !] \psi|[\varphi ?] \psi|[?] \varphi \mid[!] \varphi$.

\footnotetext{
${ }^{5}$ The composition principle also fails in $P A L$ with protocols, our topic in Section 6 .

${ }^{6}$ This Fact is not a big obstacle. We could easily extend our language with multiple questions, that do not just change partitions on a single-formula basis.
} 
These are interpreted by adding the following clauses to Definition 3:

Definition 8 (Interpretation) Formulas are interpreted in $M$ at $w$ by the following clauses, where models $M_{\varphi ?}, M_{\varphi !}, M_{\text {? }}$ and $M_{\text {! }}$ are as defined above:

$$
\begin{gathered}
M \models_{w}[\varphi ?] \psi \text { iff } M_{\varphi ?} \models_{w} \psi, \quad M \models_{w}[\varphi !] \psi \text { iff } M_{\varphi !} \models_{w} \psi, \\
M \models_{w}[!] \psi \text { iff } M_{!} \models_{w} \psi, \quad M \models_{w}[?] \psi \text { iff } M_{?} \models_{w} \psi,
\end{gathered}
$$

Interesting relations between knowledge, questions and answers can be expressed in our language. We can, for instance, say that a question $\psi$ ? is entailed in an epistemic-issue model when it does not change the issue structure:

$$
U(\widehat{Q} i \rightarrow[\psi ?] \widehat{Q} i)
$$

We can also say that a sequence of questions entails $\psi$ ? using:

$$
U\left(\left[\varphi_{0} ?\right] \cdots\left[\varphi_{n} ?\right] \widehat{Q} i \rightarrow\left[\varphi_{0} ?\right] \cdots\left[\varphi_{n} ?\right][\psi ?] \widehat{Q} i\right)^{7} \quad \text { for all nominals } i
$$

We can also express new notions of entailment, like, for instance, the notion of epistemic global entailment of an arbitrary announcement $\psi$ !:

$$
U(\widehat{R} i \rightarrow[\psi !] \widehat{R} i) \quad \text { for all nominals } i
$$

A small modification of this in which we relax the previous requirement of abstract global entailment can capture local compliance of answers:

$$
\left[\varphi_{0} ?\right] \cdots\left[\varphi_{n} ?\right](\psi \wedge \widehat{R} i) \rightarrow\left[\varphi_{0} ?\right] \cdots\left[\varphi_{n} ?\right][\psi !] \widehat{R} i^{8} \quad \text { for all nominals } i
$$

Moreover, our language can express basic laws of interrogative reasoning. For instance, we can say that an agent knows in advance that the effect of a question followed by its resolution leads to knowledge of the relevant issue:

$$
K[\varphi ?][!] U(K \varphi \vee K \neg \varphi)
$$

\subsection{Dynamic Logic of Informational Issues}

We have seen that effects of asking questions are not always easy to keep straight, but also, that there is an interesting structure to management operations on models. Both purposes call for a complete dynamic epistemic logic

\footnotetext{
${ }^{7}$ This generalizes standard definitions of entailment restricted to factual questions.

${ }^{8}$ Again, this generalizes notions of compliance restricted to propositional formulas. 
of questions. Satisfaction and validity are defined as before. The dynamic epistemic logic of questioning is the set of all semantic validities:

$$
\mathbf{D E L}_{\mathbf{Q}}=\left\{\varphi \in \mathcal{L}_{\mathbf{D E L}_{\mathbf{Q}}}(\mathrm{P}, \mathrm{N}): \vDash \varphi\right\}
$$

We introduce a proof system by adding the reduction axioms below to the earlier proof system $E L_{Q}$ for the static fragment of the logic.

What follows is a list of mostly operator commutation laws, plus clauses with crucial shifts (items $6,7,12,19,20,28$ ). This reflects the workings of our semantics of information and issue management:

Definition 9 (Reduction Axioms) The proof system $D E L_{Q}$ extends the earlier static logic $E L_{Q}$ by the following reduction axioms and inference rule:

1. $[\varphi$ ?] $a \leftrightarrow a$ (Asking \& Atoms)

2. $[\varphi ?] \neg \psi \leftrightarrow \neg[\varphi$ ?] $\psi$ (Asking \& Negation)

3. $[\varphi$ ?] $(\psi \wedge \chi) \leftrightarrow[\varphi$ ?] $\psi \wedge[\varphi$ ?] $\chi$ (Asking \& Conjunction)

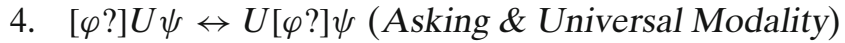

5. $[\varphi$ ?] $K \psi \leftrightarrow K[\varphi$ ?] $\psi$ (Asking \& Knowledge)

6. $[\varphi$ ?] $R \psi \leftrightarrow(\varphi \wedge R(\varphi \rightarrow[\varphi ?] \psi)) \vee(\neg \varphi \wedge R(\neg \varphi \rightarrow[\varphi ?] \psi))$ (Asking\&Resolution)

7. $[\varphi$ ?] $Q \psi \leftrightarrow(\varphi \wedge Q(\varphi \rightarrow[\varphi ?] \psi)) \vee(\neg \varphi \wedge Q(\neg \varphi \rightarrow$ $[\varphi ?] \psi))$ (Asking\&Partition)

8-11. The same as items 1 to 4 with [!] instead of [ $\varphi$ ?].

12. $[!] K \varphi \leftrightarrow R[!] \varphi$ (Resolving \& Knowledge)

13. $[!] R \varphi \leftrightarrow R[!] \varphi$ (Resolving \& Resolution)

14. [!] $Q \varphi \leftrightarrow Q[$ ! ] $\varphi$ (Resolving \& Partition)

15-18. The same as items 1 to 4 with [ $\varphi$ !] instead of [ $\varphi$ ?].

19. $[\varphi !] K \psi \leftrightarrow(\varphi \wedge K(\varphi \rightarrow[\varphi !] \psi)) \vee(\neg \varphi \wedge K(\neg \varphi \rightarrow$ $[\varphi !] \psi))(\text { Answer \& Knowledge })^{9}$

20. $[\varphi !] R \psi \leftrightarrow(\varphi \wedge R(\varphi \rightarrow[\varphi !] \psi)) \vee(\neg \varphi \wedge R(\neg \varphi \rightarrow$ $[\varphi !] \psi))($ Answer \& Resolution)

21. $[\varphi !] Q \psi \leftrightarrow Q[\varphi !] \psi$ (Announcement \& Partition)

22-25. The same as items 1 to 4 with [?] instead of [ $\varphi$ ?].

26. [?]K$\varphi \leftrightarrow K[$ ? ] $\varphi$ (Refining \& Knowledge)

27. [?] $R \varphi \leftrightarrow R[$ ? ] $\varphi$ (Refining \& Resolution)

28. [?] $Q \varphi \leftrightarrow R[$ ? ] $\varphi$ (Refining \& Partition)

29. From $\varphi$ infer $\square \varphi$, for $\square \in\{[\cdot ?],[\cdot !],[!],[?]\}$ (Necessitation)

We write $\vdash_{D E L_{Q}} \varphi$ if $\varphi$ is provable in the proof system $D E L_{Q}$.

Theorem 2 (Soundness) The reduction axioms in $D E L_{Q}$ are sound.

\footnotetext{
${ }^{9}$ If we assume truthfulness as a precondition of executing an announcement action this axiom (and other ones with a similar structure) does not need the right disjunct and will correspond to the standard DEL axioms for announcement.
} 
Proof By standard modal arguments. We discuss two cases. Asking \& Partition explains how questions refine a partition:

$$
[\varphi ?] Q \psi \leftrightarrow(\varphi \wedge Q(\varphi \rightarrow[\varphi ?] \psi)) \vee(\neg \varphi \wedge Q(\neg \varphi \rightarrow[\varphi ?] \psi))
$$

Assume that $M \models_{w}[\varphi$ ? $] Q \psi$, then we also have $M_{\varphi \text { ? }} \models_{w} Q \psi$. If $M \models_{w} \varphi$, the new issue relation locally refined the old one to $\varphi$-worlds, and hence we get the left-hand disjunct on the right. The other case yields the right-hand disjunct. The preceding explanation also works in the opposite direction.

Our second illustration (Resolving \& Knowledge) shows how resolution changes knowledge making crucial use of our intersection modality:

$$
[!] K \varphi \leftrightarrow R[!] \varphi
$$

$M \models_{w}[!] K \varphi$ is equivalent to $M_{!} \models_{w} K \varphi$, which is equivalent to $\forall v \in W_{\text {! }}$ : $w \sim$ ! $v$ implies $M_{!} \models_{v} \varphi$. As $\sim_{!}=\sim \cap \approx$, the semantics of our dynamic modality tells us that $\forall v \in W: w(\sim \cap \approx) v$ implies $M \models_{v}[!] \varphi$, which is equivalent to $M \models_{w} R[!] \varphi$, as desired.

Theorem 3 (Completeness of $\mathbf{D E L} \mathbf{L}_{\mathbf{Q}}$ ) For every formula $\varphi \in \mathcal{L}_{\mathbf{D E L}_{\mathbf{Q}}}(\mathrm{P}, \mathrm{N})$ :

$$
\models \varphi \quad \text { if and only if } \vdash_{D E L_{Q}} \varphi \text {. }
$$

Proof This is a standard $D E L$-style translation argument. Working inside out, the reduction axioms translate dynamic formulas into corresponding static ones. At the end, completeness for the static base logic is invoked. ${ }^{10}$

\subsection{Discussion}

So far we have given a logic of information and questions in standard $D E L$ style. This calculus can derive many further principles, for instance:

The following formula is provable for all factual $\varphi: \varphi \rightarrow[\varphi$ ?][!] $K \varphi$.

Proof ${ }^{11}$

$$
\begin{aligned}
& 1 \varphi \rightarrow(\varphi \wedge R(\varphi \rightarrow \varphi)) \vee(\neg \varphi \wedge R(\neg \varphi \rightarrow \varphi)) \quad P C \\
& 2 \varphi \rightarrow(\varphi \wedge R(\varphi \rightarrow[\varphi ?] \varphi)) \vee(\neg \varphi \wedge R(\neg \varphi \rightarrow[\varphi \text { ? }] \varphi)) \quad \text { Factual } \varphi \\
& 3 \varphi \rightarrow[\varphi \text { ? }] R \varphi \quad A k \& R \\
& 4 \varphi \rightarrow[\varphi \text { ? }] R[!] \varphi \quad \text { Factual } \varphi \\
& 5 \varphi \rightarrow[\varphi ?][!] K \varphi \quad R s \& K
\end{aligned}
$$

\footnotetext{
${ }^{10}$ In more detail, one needs a proof rule of Replacement of Provable Equivalents which is sound in our system. Note that this is not a Substitution Rule, which fails in DEL.

${ }^{11}$ Here and elsewhere, we need an earlier observation, now restated as: Fact: For factual formulas $\varphi$, with $q$ ranging over management actions, $[q] \varphi \leftrightarrow \varphi$ is provable. One uses the Action \& Atoms axioms for the base case and the Action \& Negation or Action \& Conjunction axioms for the inductive steps.
} 
Fig. $5 \varphi=Q \neg K p$

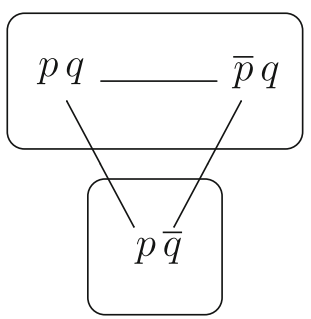

Several steps here depend on $\varphi$ being factual. They can fail otherwise as shown in Fig. 5.

The complex formula $Q \neg K p$ is true initially in every world of the model, but this is not the case anymore after a $\varphi$ ? question plus a resolution action [!].

Such changes are not always easy to keep straight, but our logic keeps track of the present, and even more complex cases. But our analysis really shows its power (compared with other approaches to questions) when we consider multi-agent scenarios and protocols for investigation. These extensions will be taken up in the next two sections.

However, we end with a remaining issue right at the present level:

Hidden Validities Like with $P A L$, the current axiomatization leave unfinished business. While reduction axioms work on a formula-by-formula basis, they need not describe the general schematic laws of the system, such as the earlier composition law for consecutive assertions, that hold under arbitrary substitutions of formulas for proposition letters. ${ }^{12}$ This deficit becomes even more urgent here, however, similar techniques can be applied in the present setting. We saw that our model-changing operations of issue management had a nice algebraic structure. For instance, it is easy to see that resolving is idempotent and commutes with refinement:

$$
! ; !=! \quad \text { and } \quad ! ; ?=? ; !
$$

But our axiomatization for $\mathbf{D E L}_{\mathbf{Q}}$ does not state such laws explicitly, since, by working only from innermost occurrences of dynamic modalities, the completeness argument needed no recursion axioms with stacked modalities like [!] [!]. Yet this sort of sequential information is obviously of great interest for a logic of issue management, and thus, we end with an

Open Problem Find a complete description for the schematic validities of our dynamic logic of issue management.

\footnotetext{
${ }^{12}$ The above reduction axioms for atoms typically lack this substitution property-though most complex reduction axioms do. For $P A L$, the long-standing open problem whether schematic validity is decidable has been solved in the affirmative in [16].
} 


\section{Multi-Agent Scenarios: Public Versions}

Questions typically involve more than one agent-and hence our restriction to single agents so far misses much of the action. In the following sections we will study multi-agent question scenarios. This is done in two stages: public questions first, and after that, questions with different informational powers for different agents.

\subsection{Static Multi-Agent Logic of Information and Issues}

The first step is routine. A static language and semantics with many agents follow entirely standard lines, taking $A$ to be a set of agents. First we modify Definition 1 to get richer epistemic issue models:

Definition 10 (Epistemic Issue Model) An epistemic issue model is a structure $M=\langle W, \stackrel{a}{\sim}, \stackrel{a}{\approx}, V\rangle$ where $W$ and $V$ are as in Definition 1 and $a \in A$ :

- $\stackrel{a}{\sim}$ is an equivalence relation on $W$ (epistemic indistinguishability),

- $\stackrel{a}{\approx}$ is an equivalence relation on $W$ (agent-issue equivalence).

Definition 11 (Static Language) The language $\mathcal{L}_{\mathbf{E L} \mathbf{Q}_{\mathbf{m}}}(\mathrm{P}, \mathrm{N}, \mathrm{A})$ has a new countable set of labels representing agents $\mathrm{A}$, with $a \in \mathrm{A}$. Its formulas are defined by the following inductive syntax rule:

$$
i|p| \perp|\neg \varphi|(\varphi \wedge \psi)|U \varphi| K_{a} \varphi\left|Q_{a} \varphi\right| R_{a} \varphi
$$

Existential modalities $\widehat{K}_{a}, \widehat{Q}_{a}$ and $\widehat{R}_{a}$ are again defined as usual.

Definition 12 (Interpretation) Formulas are interpreted in models $M$ at worlds $w$ by recursion using the same clauses as Definition 3 for atoms, Boolean and universal combinations, plus the following indexed modal ones:

$$
\begin{aligned}
& M \models_{w} K_{a} \varphi \text { iff for all } v \in W: w \stackrel{a}{\sim} v \text { implies } M=_{v} \varphi, \\
& M \models_{w} Q_{a} \varphi \text { iff for all } v \in W: w \stackrel{a}{\approx} v \text { implies } M=_{v} \varphi, \\
& M \models_{w} R_{a} \varphi \text { iff for all } v \in W: w(\stackrel{a}{\sim} \cap) v \text { implies } M=_{v} \varphi .
\end{aligned}
$$

This language can make agent-dependent distinctions. E.g., it can say that a fact $\varphi$ is an issue for some agents and not for others:

$$
U\left(Q_{a} \varphi \vee Q_{a} \neg \varphi\right) \wedge \neg U\left(Q_{b} \varphi \vee Q_{b} \neg \varphi\right)
$$

It can also say that one agent's information is linked with issues for another:

$$
\widehat{K}_{a} \varphi \rightarrow R_{b} \psi \quad \text { or } \quad K_{b} \psi \rightarrow\left(\varphi \wedge \widehat{Q}_{a} \neg \varphi\right)
$$


It can also describe much more deeply intertwined knowledge and issues.

Next, we need to bring out the dynamics. We first consider public actions as before. These lead to an immediate generalization of earlier results:

Theorem 4 (Completeness for Public Multi-Agent $\mathbf{D E L} \mathbf{L}_{\mathbf{Q}}$ ) The logic of multiagent epistemic questions is completely axiomatizable.

Proof The proof consists in suitable agent labeling of earlier reduction axioms. We just illustrate this routine generalization with the indexed versions of two previous axioms (Answer \& Knowledge and Asking \& Partition):

$$
\begin{aligned}
& {[\varphi !] K_{a} \psi \leftrightarrow\left(\varphi \wedge K_{a}(\varphi \rightarrow[\varphi !] \psi)\right) \vee\left(\neg \varphi \wedge K_{a}(\neg \varphi \rightarrow[\varphi !] \psi)\right),} \\
& {[\varphi ?] Q_{a} \psi \leftrightarrow\left(\varphi \wedge Q_{a}(\varphi \rightarrow[\varphi ?] \psi)\right) \vee\left(\neg \varphi \wedge Q_{a}(\neg \varphi \rightarrow[\varphi ?] \psi)\right)}
\end{aligned}
$$

Remark on Groups Our static language only contains issue modalities for single agents. But like in epistemic logic, groups of agents have inquiry-related behavior of their own. Thus, a full treatment would require group notions of information (such as common and distributed knowledge), and operators for 'collective issues' owned by groups rather than individuals.

\subsection{Agent-Specific Questions and Preconditions}

So far, we only had impersonal public questions among many agents. Now we must consider real questions, as asked by one agent to another. As everywhere in this paper, we restrict attention to propositional questions: ${ }^{13}$

$$
b \text { asks } a \text { : 'Is } \varphi \text { the case?' }
$$

Thus, real 'agency' enters when we consider the structure of question acts. But does this call for new management actions?

Consider an analogy with public announcement logic $P A L$. Real speech acts of announcement, too, are agent-relative: ' $b$ tells $a$ that $\varphi$ '. But one usually performs a reduction here: saying that $\varphi$ is treated as an impersonal public announcement of the conversational precondition of this event.

What that precondition is may depend on one's pragmatic theory. But it will usually involve agent-oriented facts such as:

' $b$ knows $\varphi$ ', and perhaps even: ' $b$ believes that $a$ does not know that $\varphi$ '.

\footnotetext{
${ }^{13}$ Extension to other types, such as Wh-questions, seems straightforward to us, though it would need a predicate-logical version of dynamic-epistemic logics.
} 
Thus, agents come in through impersonal public announcement of agentdependent preconditions. ${ }^{14}$ Some common multi-agent preconditions are:

1. ' $b$ asks $\varphi$ ' presupposes $\neg K_{b} \varphi \wedge \neg K_{b} \neg \varphi$ : that is, the questioner must not know the answer to the question she asks,

2. ' $b$ asks $\varphi$ to $a$ ' presupposes $\widehat{K}_{b}\left(K_{a} \varphi \vee K_{a} \neg \varphi\right)$ : that is, the questioner must consider it possible that the questionee knows the answer.

These make sense for truly informative Gricean questions, and there may be other preconditions. For instance, asking a question usually suggests that one wants to know the answer. But of course, questions come in many varieties. Rhetorical questions by a teacher to the students do not convey that the teacher does not know the answer, and they definitely do not suggest that the teacher is under any illusions whether the students know the answer.

Such typology of questions: 'plain', 'rhetorical', 'Socratic', is not a task for logic, however. Indeed, a language of the sort we have developed here can formulate lots of different preconditions, and logic is neutral on any choice between them. ${ }^{15}$ The only thing our logic needs to do at this stage is keep track of possible preconditions for agent-related questioning actions

$$
\varphi ?_{a}^{b}: \quad \text { ' } b \text { asks } \varphi \text { to } a \text { '. }
$$

We assume that some precondition $\operatorname{pre}\left(\varphi ?_{a}^{b}\right)$ is given for this, and likewise, a $\operatorname{pre}\left(\varphi !{ }_{a}^{b}\right)$ for an act of $b$ 's saying that $\varphi$ to $a$. Then we can formulate management actions and their dynamic logic along standard lines:

Definition 13 (Questioning Actions) Executing $\varphi ?_{a}^{b}$ in $M$ gives a new model $M_{\varphi ? a}=\left\langle W_{\varphi ? a}, \stackrel{c}{\sim}{ }_{\varphi ? a}, \stackrel{c}{\sim}_{\varphi ?_{a}^{? b}}, V_{\varphi ? a}\right\rangle$. Likewise, $\varphi !_{a}^{b}$ leads to a new model $M_{\varphi ! b}=$ $\left\langle W_{\varphi ! a}, \stackrel{c}{\sim} \underset{\varphi ! a}{,}, \stackrel{c}{\approx} \sigma_{! ! a}^{b}, V_{\varphi ! a}\right\rangle$. 'Resolution' and 'refinement' are as before:

$$
\begin{aligned}
& W_{\varphi ?_{a}^{b}}=W \\
& \stackrel{c}{\sim}_{\varphi ? b}=\stackrel{c}{\sim} \cap \stackrel{\operatorname{pre}\left(\varphi ? ?_{a}^{b}\right)}{=} \\
& \stackrel{c}{\sim}_{\varphi ? b}=\stackrel{c}{\approx} \cap \stackrel{\varphi}{=}_{M} \\
& V_{\varphi ? a}=V
\end{aligned}
$$

$$
\begin{aligned}
& W_{\varphi ! a}=W \\
& {\underset{c}{\varphi ! b}}_{a}^{c}=\stackrel{c}{\sim} \cap \stackrel{\operatorname{pre}(\varphi ! b)}{=} \cap \cap \stackrel{\varphi}{=}_{M} \\
& {\underset{\sim}{c}}_{\varphi ! b}=\stackrel{\sim}{\sim} \\
& V_{\varphi ! a}=V
\end{aligned}
$$

\footnotetext{
${ }^{14}$ The same is true if we add further agent-dependent aspects of taking what is said, such as the reliability that $a$ assigns to the source $b$. This may be reflected in degrees of 'softness' of the signal $\varphi$, as in dynamic logics of belief revision (cf. [26]). We will not pursue this here, since reliability seems to play less of a role in the process of raising issues. But similar phenomena would come up if one gave speakers 'authority' in raising issues.

${ }^{15}$ Our further issue management actions like resolution, refinement, or their parallel execution may also have multi-agent preconditions. We do not pursue these here.
} 


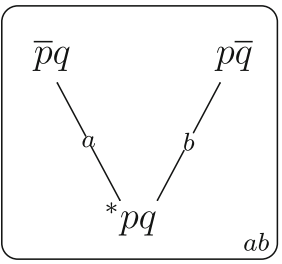

$\otimes$

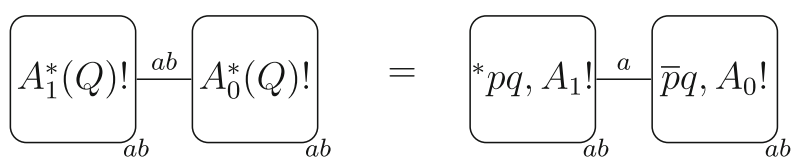

Fig. 6 Dealing with agency using epistemic preconditions when asking agent dependent question $(p \wedge q)$ ? in multi-agent epistemic-issue environment

In this definition, assertions refine the epistemic relation by means of their preconditions and also by their content. In our link-cutting version, this refines the given relation into 4 equivalence classes. ${ }^{16}$ Questions act differently: they refine the issue relation with their content, but they also refine the epistemic relation through the information in their precondition. Our clauses capture these ideas formally.

Example 1 (Solving a Question by Raising It) This multi-agent semantics can deal with interesting phenomena. For instance, the combination of preconditions and issue change can have surprising effects which go beyond mere refinement of the issue relation. In particular, agents can solve a question by raising it. Consider the example in Fig. 6 where by asking a question $a$ simultaneously gives $b$ the answer to the very issue that he raises. Here agent $a$ asks the question $Q=(p \wedge q)$ ? to agent $b$, with the standard yes/no answers $\left\{A_{0}, A_{1}\right\}$ and the following combined epistemic preconditions pre $(Q)=\neg\left(K_{a}(p \wedge q) \vee \neg K_{a}(p \wedge q)\right) \wedge \widehat{K}_{a}\left(K_{b}(p \wedge q) \vee\right.$ $\left.K_{b} \neg(p \wedge q)\right)$, pre $\left(A_{1}(Q)\right)=p \wedge q \wedge \operatorname{pre}(Q)$, and pre $\left(A_{0}(Q)\right)=\neg(p \wedge q) \wedge$ $\operatorname{pre}(Q)$. Much more complex scenarios can be dealt with, but this will give the flavour.

\subsection{Dynamic Language, Logic, and Some Design Issues}

Now we can add a dynamic language as usual, and interpret it over multi-agent epistemic issue models. This proceeds just as before in Section 3.3.

Theorem 5 There is a complete dynamic logic with reduction axioms for public multi-agent questions extending the single-agent one of Section 3.4 .

\footnotetext{
${ }^{16}$ With world-eliminating announcements, we could just restrict the domain to the worlds satisfying one formula: the conjunction of the content and the precondition.
} 
Proof We only display here the three key reduction axioms:

(Asking \& Knowledge), where $\chi=\operatorname{pre}\left(\varphi ?_{a}^{b}\right)$ : $\left[\varphi ?_{a}^{b}\right] K_{c} \psi \leftrightarrow\left(\chi \wedge K_{c}\left(\chi \rightarrow\left[\varphi ?_{a}^{b}\right] \psi\right)\right) \vee\left(\neg \chi \wedge K_{c}\left(\neg \chi \rightarrow\left[\varphi ?_{a}^{b}\right] \psi\right)\right)$, (Asking \& Intersection): $\left[\varphi ?_{a}^{b}\right] R_{c} \psi \leftrightarrow \bigvee_{i}\left\{\chi_{i} \wedge R_{c}\left(\chi_{i} \rightarrow\left[\varphi ?_{a}^{b}\right] \psi\right)\right\}$, $\chi_{i} \in\left\{\operatorname{pre}\left(\varphi ?_{a}^{b}\right) \wedge \varphi, \neg \operatorname{pre}\left(\varphi ?_{a}^{b}\right) \wedge \varphi, \operatorname{pre}\left(\varphi ?_{a}^{b}\right) \wedge \neg \varphi, \neg \operatorname{pre}\left(\varphi ?_{a}^{b}\right) \wedge \neg \varphi\right\}$ (Announcement \& Knowledge): $\left[\varphi !_{a}^{b}\right] K_{c} \psi \leftrightarrow \bigvee_{i}\left\{\chi_{i} \wedge K_{c}\left(\chi_{i} \rightarrow\left[\varphi !_{a}^{b}\right] \psi\right)\right\}$, $\chi_{i} \in\left\{\operatorname{pre}\left(\varphi ! a_{a}^{b}\right) \wedge \varphi, \neg \operatorname{pre}\left(\varphi ! !_{a}^{b}\right) \wedge \varphi, \operatorname{pre}\left(\varphi !_{a}^{b}\right) \wedge \neg \varphi, \neg \operatorname{pre}\left(\varphi !_{a}^{b}\right) \wedge \neg \varphi\right\}$

The overall completeness argument runs exactly like before.

A similar completeness theorem can be proved with slightly modified axioms for the usual world-eliminating announcement actions.

But modified dynamics make sense, too. Our treatment of questions amounts to a parallel operation on epistemic issue models: we change epistemic accessibility and the issue relation at the same time, using the precondition of the question and its content, respectively. An alternative might be to do this sequentially - but then, for complex questions, updating with the precondition might change the model in a way that affects the subsequent change in the issue relation. The two approaches are not the same and the parallel version is closer to the intuitive effect of a question. ${ }^{17}$

\section{Product Update for Multi-Agent $D E L_{Q}$}

Now we move to the full range of epistemic dynamics: informational actions that involve different informational powers for agents, ranging from differences in public abilities to privacy and hiding. These same phenomena make sense with questions and issue management. There may be differences in agents' powers, and questions can be private just as well as observations. And mixtures can occur, too: a question may be public, but the answer partly private, or an answer may be public, and the question private. Indeed, in the setting of social interaction or even scientific inquiry, subtle distinctions of this sort make sense.

We assume that the reader is familiar with the basics of product update for static epistemic models $M$ with so-called 'event models' $E$, the dynamicepistemic mechanism appropriate to this setting (cf. the classic paper [5]). ${ }^{18} \mathrm{In}$

\footnotetext{
${ }^{17}$ Moreover, our system has some independent technical interest, adding a new program operation of parallel execution to dynamic-epistemic logic.

${ }^{18}$ Here are the basics in a nutshell. An event model E consists of a set of relevant events related by epistemic uncertainty links that encode agents' observational powers. Moreover, each event comes with a 'precondition' stating just when it can occur: these drive the information flow when events are observed. This shows in forming a product model $\mathrm{M} \times \mathrm{E}$ consisting of all pairs $(s, e)$ of old worlds $s \in \mathrm{M}$ that satisfy the precondition for event $e$. The new knowledge of agents is encoded in the new uncertainty relation between pairs, which goes by the 'product rule': $(s, e) \sim(t, f)$ iff $s \sim e$ and $e \sim f$. Finally, the valuation for proposition letters at $(s, e)$ remains the same as that as $s:$ at least in the simple $D E L$ systems that we discuss here, there is no factual change.
} 


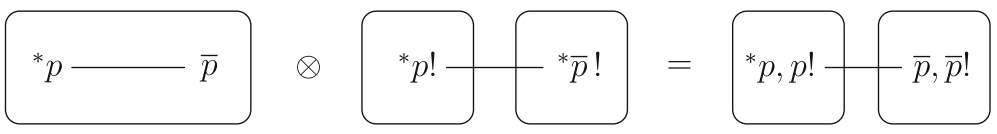

Fig. 7 Question $p$ ? in a single-agent epistemic-issue structure

this section, we will merely sketch how this method can be extended to include issue management.

The main idea is simply this: we add issue relations to static epistemic models, but also to epistemic event models. It then turns out that the usual product update rule makes sense for both kinds of relation-though there are interesting new points of interpretation and application.

\subsection{A Simple Motivating Example}

Before defining a formal counterpart to product update in our new setting, let us discuss what needs to be done, starting from the basic type of question that we have studied before.

Consider the simple scenario depicted in Fig. 7: a public Yes/No question is asked in a single-agent structure. As the relevant events, we have chosen two abstract epistemic signals that model the two possible answers to the question. The epistemic uncertainty relation connects these two events, since the agent does not yet know which one will actually occur. By contrast, the issue relation chosen in this event model does distinguish the answers.

As indicated in Fig. 7, is easy to see, even without formal definitions, that product update produces exactly the new model that we defined earlier in Section 3. The same is true for other examples that we discussed earlier.

Simple as this proposal looks, it shows some divergences from $D E L$.

Observation Versus Prediction Uncertainty In $P A L$ and $D E L$, epistemic indistinguishability between events represents observational uncertainty for agents. In the present setting, there is no observation of possible future answers yet, and hence the epistemic relation rather models predictive uncertainty. ${ }^{19}$ Likewise, the issue relation changes its intuitive meaning. The point is not that answer events are issues by themselves, but rather, the issue relation in an event model makes agents aware of possibilities for resolving questions in a future answering move. ${ }^{20}$ We could call this the highlighting function of the issue relation.

\footnotetext{
${ }^{19}$ Likewise, in games, 'future ignorance' about a next move is not lack of observational power about moves so far, but uncertainty about what will happen.

${ }^{20}$ The issue of awareness also has syntactic aspects that we discuss briefly later on.
} 
Distinguished Sets of Worlds While the initial epistemic model usually has one actual world, there is no such distinguished world in our question-event model. No possible answer is distinguished at this stage. Therefore, we will use a version later where models can have sets of distinguished worlds or sets of distinguished events - a generalization that has also been considered for $D E L$ itself. An answer event becomes distinguished once it has actually been given: we will return to representation of answers in the next subsection.

Two Kinds of Preconditions Answer events have the precondition that their content is true. But in addition, we also saw that questions themselves may have preconditions, such as ignorance of the answer. Should these be represented in the event model? One option is to have the precondition as a separate announcement before the product update, another option (less intuitive, but sometimes convenient) would be to copy the precondition of the whole question into the preconditions for each answer.

With this simplest setting in place, we turn to more complex cases beyond what most logics of questions can handle. These include:

- Mixtures of prediction uncertainty and observation uncertainty. An agent knows that some question is asked, but does not know exactly what that question is: it could be either $p$ ? or $q$ ?

- Genuine privacy. An agent hears the question $p$ ?, but another agent thinks that nothing happened.

We now formulate a mechanism that can deal with such scenarios, that are ubiquitous in daily life and our natural communicative practice.

\subsection{Product Update for Questions}

It is time for a precise model that can account for the above phenomena. In order to do this, we use the following event models:

Definition 14 An action structure $\mathrm{E}=\langle E, \stackrel{a}{\sim}, \stackrel{a}{\approx}$, pre $\rangle$ has the following:

- $\quad E$ is a set of events (possible future answer events),

- $\stackrel{a}{\sim}$ are equivalence relations on $E$ (prediction uncertainty),

- $\stackrel{a}{\approx}$ are equivalence relations on $E$ (issue highlight relation),

- pre: $E \rightarrow \mathcal{L}_{\mathbf{E L Q}_{\mathrm{m}}}$ is a precondition function mapping events $e \in E$ to formulas of the relevant epistemic issue language $\mathcal{L}_{\mathbf{E L Q}}{ }^{21}$

\footnotetext{
${ }^{21}$ There is a 'parametrization' here to some appropriate language for specifying preconditions. This language can be a propositional base logic of factual assertions, the richer static epistemic issue language of Section 2.2, or even the full dynamic language $\mathcal{L}_{\mathbf{E L}} \mathbf{Q}_{\mathbf{m}}$ of Section 5.4 below. The latter choice involves a slightly delicate issue of mutual recursion, but since it is similar to that for $D E L$ in general, we do not discuss it here.
} 

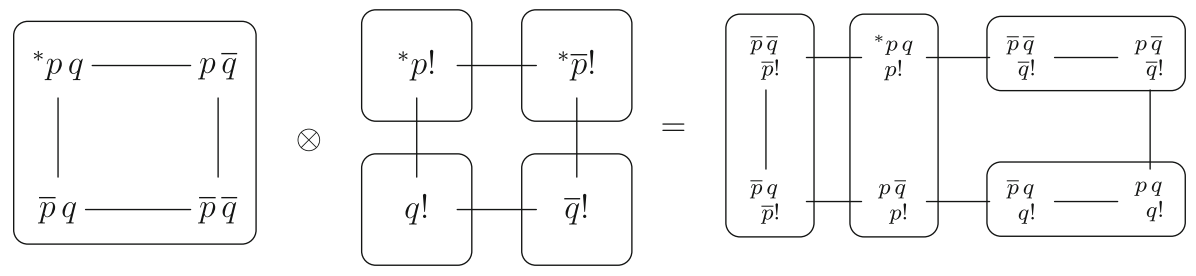

Fig. 8 Intuitive dynamics for asking more indistinguishable questions

Here is how these structures transform given epistemic issue models:

Definition 15 For $M=(\langle W, \stackrel{a}{\sim}, \stackrel{a}{\approx}, V\rangle, w)$, and $\mathrm{E}=(\langle E, \stackrel{a}{\sim}, \stackrel{a}{\approx}$, pre $\rangle, e)$ the $u p$ date product $M \otimes \mathrm{E}=M_{\otimes}^{\mathrm{E}}=\left(\left\langle W_{\otimes}, \stackrel{a}{\sim} \sim_{\otimes}, \stackrel{a}{\approx}_{\otimes}, V_{\otimes}\right\rangle,(w, e)\right)$ is defined by:

- $W_{\otimes}=\left\{(w, e)|w \in W, e \in E, M|=_{w} \operatorname{pre}(e)\right\}$,

- $\stackrel{a}{\sim} \underset{\otimes}{ }=\left\{\left((w, e),\left(w^{\prime}, e^{\prime}\right)\right) \mid w, w^{\prime} \in W, e, e^{\prime} \in E, w \stackrel{a}{\sim} w^{\prime}, e \stackrel{a}{\sim} e^{\prime}\right\}$,

- $\stackrel{a}{\approx}_{\otimes}=\left\{\left((w, e),\left(w^{\prime}, e^{\prime}\right)\right) \mid w, w^{\prime} \in W, e, e^{\prime} \in E, w \stackrel{a}{\approx} w^{\prime}, e \stackrel{a}{\approx} e^{\prime}\right\}$,

$-V_{\otimes}(w, e)=V(w) .^{22}$

Now we give some examples demonstrating how this mechanism works. We start with two earlier desiderata:

Example 2 (Uncertainty About the Question) Figure 8 depicts a scenario where a question is asked but, as far as the observing agent is concerned, the content of the question could be either $p$ or $q$.

Example 3 (Radical Privacy) Figure 9 depicts a question that is not noticed by an agent, who thinks nothing happened. This involves mistaken beliefs of agents. Hence, epistemic relations need no longer be reflexive-but as with $D E L$, this changes nothing essential in the product update mechanism.

We end with one more example, now involving two agents essentially:

Example 4 (Privacy in Multi-Agent Scenarios) Figure 10 shows how product update computes an intuitive result in a scenario where different agents have different information about the content of a public question.

For technical details, and alternative versions of the mechanism, cf. [21].

\footnotetext{
${ }^{22}$ If we need to work with sets of distinguished events, as indicated earlier, we take all pairs of an earlier distinguished world plus any answer event supported by it.
} 

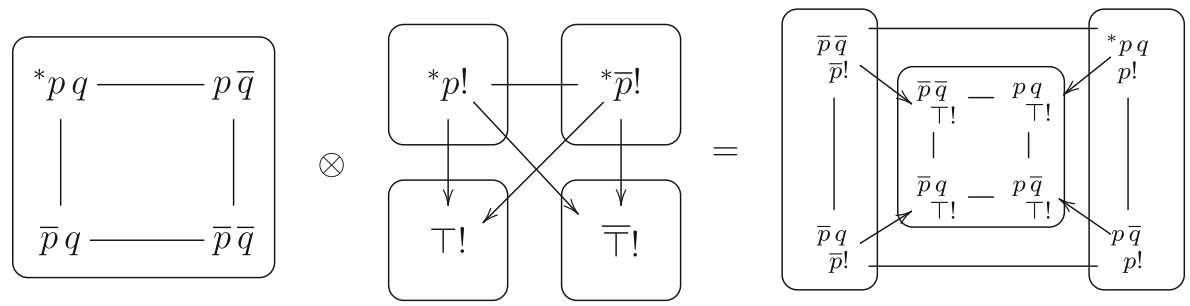

Fig. 9 Intuitive dynamics for radical privacy about questions

\subsection{Discussion}

These examples raise some further issues that we state briefly here.

Special Event Models for Questions Questions are just a particular type of event, and a more realistic treatment might capture them as a subclass of the above event models, using the earlier examples as a guide. For instance, it seems plausible that answers to observation-distinguishable questions should be prediction-distinguishable, and further constraints are easily found. We leave a fuller treatment of these issues to future investigation.

Modeling Answers as Separate Events In Section 3, answers to questions $\varphi$ ? were not modeled separately - though one might think of announcements $\varphi$ ! or $\neg \varphi$ ! as answer events. An answer might be modeled explicitly as an event model with its own presuppositions: for instance, an answerer communicates that she does know the answer. But also, answering involves selection of one possible answer as the actual one, removing the earlier prediction uncertainty, though perhaps subject to observation uncertainty. It is easy to model such scenarios with our machinery.

Further Issue Management Actions We have not discussed counterparts to our earlier actions of Resolution and Refinement (cf. Section 3) that went beyond simple questions and answers. These require modifications of our product update rule that we do not pursue here.
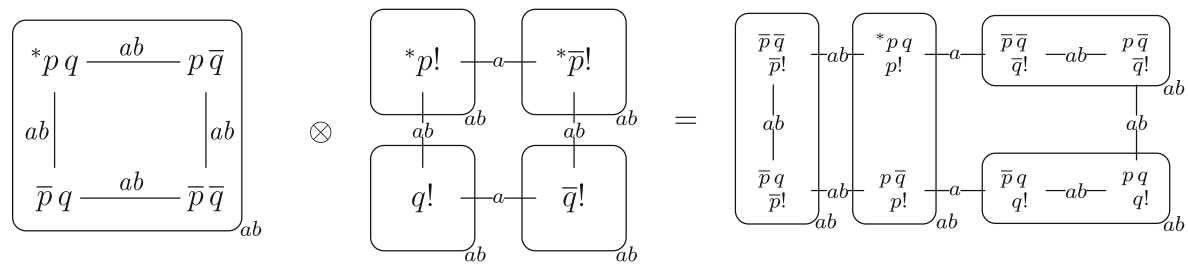

Fig. 10 Product update for a scenario in which $b$ knows that the content of the question is $p$ ? but $a$ considers that the content might have been $q$ ? 
The Role of Syntax: Formulation and Protocols Our treatment here has been semantic, identifying propositions with sets of worlds in the usual manner. Thus, the two questions $(p \vee p)$ ? and $p$ ? will have exactly the same effects, and can be considered the same. ${ }^{23}$ This ignores the role of syntactic formulation in inquiry, and more modestly, in the appropriateness of answers to a stated question. But actually, product update also suggests a more syntactic viewpoint, where the answer events are actual linguistic formulations of answers. This syntactic perspective will be developed in Section 6 below, when discussing protocols that constrain avenues of investigation.

\subsection{Complete Dynamic Logics for Product Update}

The preceding dynamics can be described in a language extending our systems from Section 3. We add dynamic modalities over event models:

Definition 16 (Dynamic Language) Formulas of the dynamic epistemic issue language $\mathcal{L}_{\mathbf{E L}} \mathbf{Q}_{\mathrm{m}}$ are constructed by the following inductive-syntax rule:

$$
i|p| \perp|\neg \varphi|(\varphi \wedge \psi)|U \varphi| K_{a} \varphi\left|Q_{a} \varphi\right| R_{a} \varphi \mid[\mathrm{E}] \varphi
$$

Definition 17 (Semantics) Formulas are interpreted with this key clause:

$$
M \models_{w}[\mathrm{E}] \varphi \quad \text { iff } \quad M \otimes \mathrm{E} \models_{w} \varphi .
$$

The resulting dynamic epistemic logic can be axiomatized by standard techniques, in terms of reduction axioms for event modalities:

Theorem 6 (Completeness) The dynamic epistemic issue logic of product update is completely axiomatizable.

Proof We merely state a few relevant principles:

(Asking \& Knowledge):

$$
[\mathrm{E} ?] K_{a} \varphi \leftrightarrow \bigvee_{e \in Q^{\mathrm{E}}}\left(\operatorname{pre}(e) \rightarrow \bigwedge_{\mathrm{E} ? \stackrel{a}{\sim} \mathrm{E}^{\prime} ?} K_{a}\left[\mathrm{E}^{\prime} ?\right] \varphi\right),
$$

(Asking \& Partition):

$$
[\mathrm{E} ?] Q_{a} \varphi \leftrightarrow \bigvee_{e \in Q^{\mathrm{E}}}\left(\operatorname{pre}(e) \rightarrow \bigwedge_{\mathrm{E} ? \stackrel{a}{\sim} \mathrm{E}^{\prime} ?} Q_{a}\left[\mathrm{E}^{\prime} ?\right] \varphi\right)
$$

\footnotetext{
${ }^{23}$ Technically, the reason is that our logic cannot detect differences in models up to epistemic-issue bisimulation.
} 
(Answering \& Knowledge):

$$
[\mathrm{E} !] K_{a} \varphi \leftrightarrow \bigwedge_{\mathrm{E} ! \stackrel{a}{\sim} \mathrm{E}^{\prime} !} R_{a}\left[\mathrm{E}^{\prime} !\right] \varphi,{ }^{24}
$$

We have shown how the technique of product update allows for a drastic extension of modeling power for inquisitive scenarios. Our treatment is by no means complete, ${ }^{25}$ but it may show how current 'logics of questions' can undergo the same broadening that epistemic logic has experienced in DEL.

\section{Temporal Protocols for Inquiry}

Our final topic is another recent theme from dynamic-epistemic logic, viz. longer-term temporal perspective and 'procedural information' (cf. [17]).

Single announcements usually only make sense in a longer-term temporal perspective of some ongoing informational process: a conversation, an experimental protocol, a learning mechanism, and so on. To make this procedural information explicit, [29] introduces protocols into dynamic-epistemic logic. This results in modified versions of the public announcement logic $P A L$, which now encode procedural as well as factual and epistemic information. ${ }^{26}$

But the same considerations apply to questions, perhaps even more so. Not everything can be asked, because of social convention, or limitations on our measuring apparatus, or financial resources. Thus, it makes sense to adapt our dynamic logics to a protocol setting, and we will show how this can be done, resulting in a more realistic theory of inquiry.

We first present several settings where temporal restrictions on questions make sense. We do this in some detail, to show a crucial dimension of questions and inquiry that is often ignored in the literature.

Restrictions on Types of Questions Clearly, there are strong restrictions on the kinds of question that can be asked in realistic inquiry. We measure 'little things' in science, such as readings of instrument panels, and try to get insights

\footnotetext{
${ }^{24}$ Here we use the following notation: $\mathrm{E}$ ? $\stackrel{a}{\sim} \mathrm{E}^{\prime} ?$ if and only if $E_{\mathrm{E} ?}=E_{\mathrm{E}^{\prime}}, \stackrel{a}{\sim}{ }_{\mathrm{E}}$ ? $=\stackrel{a}{\sim} \mathrm{E}^{\prime} ?, \stackrel{a}{\sim} \mathrm{E}_{\mathrm{E}}=$ $\stackrel{a}{\approx}_{\mathrm{E}^{\prime} ?}$, pre $\mathrm{E}_{\mathrm{E} ?}=\operatorname{pre}_{\mathrm{E}^{\prime} \text { ? }}$ and $\forall e, e^{\prime} \in Q_{\mathrm{E}^{*}}^{*} \cup Q_{\mathrm{E}^{\prime} ?}^{*}: e \stackrel{a}{\sim} e^{\prime} \& e \stackrel{a}{\approx} e^{\prime} \Leftrightarrow e=e^{\prime}$, with $Q_{\mathrm{E} \text { ? }}^{*}$, distinguished set of events representing the actual question, as discussed before, and, $E ! \stackrel{a}{\sim} E^{\prime} !$ if and only if $E_{\mathrm{E} !}=E_{\mathrm{E}^{\prime} !}, \stackrel{a}{\sim} \mathrm{E}_{\mathrm{E}}=\stackrel{a}{\sim} \mathrm{E}^{\prime} !, \stackrel{a}{\sim} \mathrm{E}_{\mathrm{E} !}=\stackrel{a}{\sim} \mathrm{E}^{\prime} !$ pre $\mathrm{E} !=$ pre $\mathrm{E}^{\prime} !$ and $e_{\mathrm{E} !}^{*} \stackrel{a}{\sim} e_{\mathrm{E}^{\prime} !}^{*}$.

${ }^{25}$ See $[21,28]$ for technical improvements of the approach sketched here, with more complex questioning and resolution action models capturing further realistic features. These include a precise way of lifting uncertainty between answers to indistinguishability between action models for questions, and a product update rule that takes into account both issue and uncertainty relations and their intersection. There are also various kinds of resolution actions having preconditions expressed in a language that describes the structure of the issue, thus linking answers to previous questions.

${ }^{26}$ As a technical side-effect, the original reduction axioms no longer do all the work, as procedural information may be irreducible. Completeness proofs become more complex.
} 
about larger issues. Thus, in a logic setting, we may only be able to ask about atomic questions $p$ ?, and not about very complex formulas $\varphi$ ?. The same is true in conversation: we are usually restricted to simple questions that are easy to understand. More generally, we can think of this as a logical hierarchy, from factual questions to epistemic ones ('Did you know that ...?') to complex procedural ones ('What would your sister say if I asked her what I just asked you?').

These things can be demonstrated even in the simple setting of propositional logic. Atomic questions may take more time to reach a goal than more complex ones. Figure 11 gives a very simple illustration. This figure also demonstrates another interesting aspect. Information Theory measures information bit content in terms of the number of factual Yes/No questions needed to determine the real situation-but it has no restriction to just atomic questions. Thus, one may sometimes need more atomic questions than the usual bit measure would indicate.

Procedural Constraints on Questioning Even if the available types of question are fixed, there can be further procedural restrictions, having to do with access to experimental devices.

Consider a classical mathematical 'Weighing Problem' like the following:

"You have 9 pearls, 1 lighter than the others that are all of equal weight. You can weigh only 2 times with a standard balance.

Find the lighter pearl.

The admissible questions are fixed in this protocol: we can only ask Nature for some atomic balance facts. The resulting process can be pictured in an

Fig. 11 Complex experiments versus atomic questioning

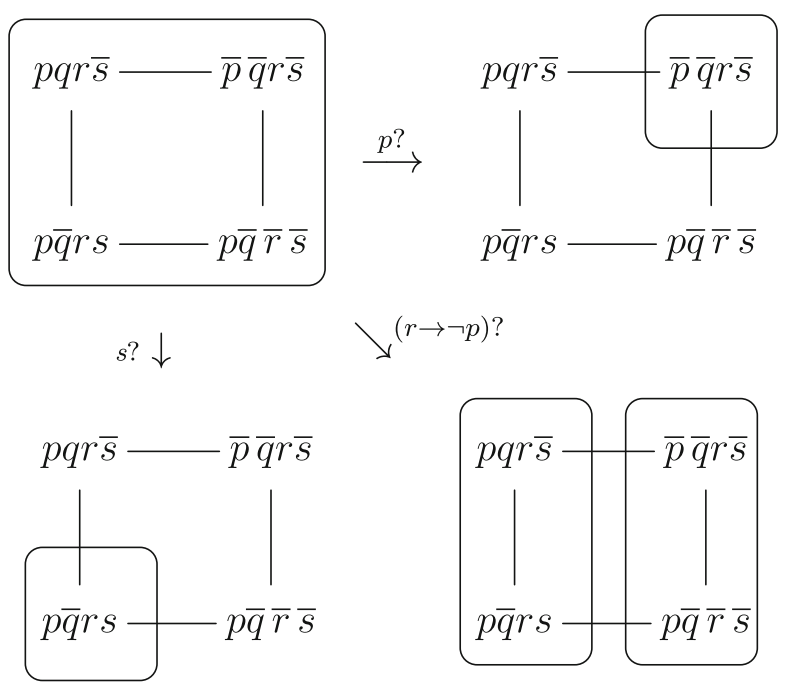




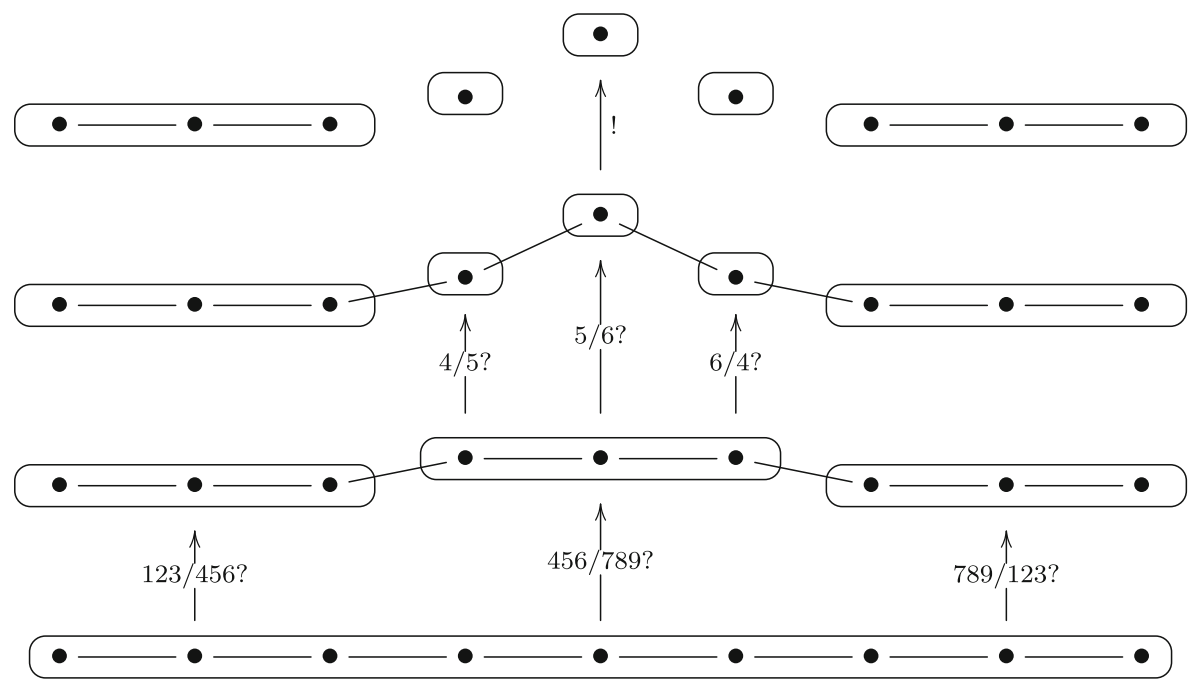

Fig. 12 Procedural restrictions in the number of available questions

'event tree' of possible histories of successive weighings. Figure 12 illustrate a few successful evolutions, but there could also be 'bad' ones. ${ }^{27,28}$

Restrictions from Epistemic Properties of Inquiry Even if types of questions and procedural restrictions are fixed, there may still be further relevant restrictions in multi-agent scenarios. In games, for instance, it might be of crucial importance in what temporal order new knowledge is obtained. If the competition finds out the actual world ahead of time, there is no incentive for sharing resources in a social experimental procedure.

All these aspects show how dynamic logics of questions suddenly get a much richer field of study than single linguistic speech acts, or information-theoretic question scenarios where anything goes.

Some Formalities The preceding examples can be formalized in an epistemic question protocol logic that borrows largely from [29] and [17]:

Definition 18 (DEL $\mathrm{D}_{\mathrm{Q}}$ Protocol) Let $\Sigma$ be an arbitrary set of epistemic events (management actions). Let $\Sigma^{*}$ be the set of finite strings over $\Sigma$ (finite histories

\footnotetext{
${ }^{27}$ For instance, asking $(7 / 8)$ ? after $(789 / 123)$ ? is a history that fails to solve the problem in two steps, and hence it would be a bad protocol.

${ }^{28} \mathrm{By}$ the way, here is the solution to this Weighing Problem: "Weigh 3 pearls against 3, leaving 3 pearls aside. If the result is equal, then weigh 2 of the the remaining 3 pearls against each other. If these are equal, then the remaining pearl is the lighter one-if not, then you know which one was lightest. If the initial result was unequal, apply the previous procedure to the lighter group of 3: weigh 2 of these, and you will know."
} 
of questioning events). A questioning protocol is a set $\mathcal{H} \subseteq \Sigma^{*}$ (containing all non-empty finite histories and all their prefixes, or every rooted sub-history, or each initial segment) such that:

$$
\text { FinPre }_{-\lambda}(\mathcal{H})=\left\{h \mid h \neq \lambda, \exists h^{\prime} \in \mathcal{H}: h \preceq h^{\prime}\right\} \subseteq \mathcal{H}
$$

Next, we can set up a formal language over these structures. Crucially, in the truth definition, dynamic modalities now state that there exists a next informational or issue action available at this stage by the current protocol whose execution makes the postcondition true.

The construction in the following definition considers only sequences $w \sigma$ such that $w$ is a world in the domain of the initial model $M$, and $\sigma, \sigma^{\prime}$ are sequences in the state dependent protocol $Q(w) ; \sigma_{n}$ denotes the sequence $\sigma$ up to its $n$-th position and $\sigma_{(n)}$ denotes the $n$-th element in the sequence.

Definition 19 ( $Q$-Generated Model) Let $M=\langle W, \sim, \approx, V\rangle$ be an arbitrary model and let $Q$ be an arbitrary $\mathrm{DEL}_{\mathrm{Q}}$-protocol over $M$ (a prefix-closed set of finite sequences of questioning events). The $Q$-Generated Model at level $n$, $M_{Q}^{n}=\left\langle W_{Q}^{n}, \sim_{Q}^{n}, \approx_{Q}^{n}, V_{Q}^{n}\right\rangle$ is defined by induction on $n$ as follows:

1. $W_{Q}^{0}=W, \quad \sim_{Q}^{0}=\sim, \quad \approx_{Q}^{0}=\approx, \quad V_{Q}^{0}=V$;

2. $\quad w \sigma \in W_{Q}^{n+1}$ iff $w \in \operatorname{dom}(M), \sigma \in Q(w), \operatorname{len}(\sigma)=n+1$, and $w \sigma_{n} \in W_{Q}^{n}$;

3. If $\sigma_{(n+1)}=\varphi$ ?, then:

(a) $\left(w \sigma, v \sigma^{\prime}\right) \in \sim_{Q}^{n+1} \quad$ iff $\quad \sigma_{(n+1)}=\sigma_{(n+1)}^{\prime}$, and $\left(w \sigma_{n}, v \sigma_{n}^{\prime}\right) \in \sim_{Q}^{n}$;

(b) $\left(w \sigma, v \sigma^{\prime}\right) \in \approx_{Q}^{n+1}$ iff $\sigma_{(n+1)}=\sigma_{(n+1)}^{\prime},\left(w \sigma_{n}, v \sigma_{n}^{\prime}\right) \in \approx_{Q}^{n},\left(w \sigma_{n}, v \sigma_{n}^{\prime}\right) \in \stackrel{\underline{\underline{\varphi}}}{=}_{M_{Q}^{n}}$;

4. If $\sigma_{(n+1)}=$ !, then:

(a) $\left(w \sigma, v \sigma^{\prime}\right) \in \approx_{Q}^{n+1} \quad$ iff $\quad \sigma_{(n+1)}=\sigma_{(n+1)}^{\prime}$, and $\left(w \sigma_{n}, v \sigma_{n}^{\prime}\right) \in \approx_{Q}^{n}$;

(b) $\left(w \sigma, v \sigma^{\prime}\right) \in \sim_{Q}^{n+1}$ iff $\sigma_{(n+1)}=\sigma_{(n+1)}^{\prime},\left(w \sigma_{n}, v \sigma_{n}^{\prime}\right) \in \sim_{Q}^{n},\left(w \sigma_{n}, v \sigma_{n}^{\prime}\right) \in \approx_{Q}^{n}$;

5. For each $a \in \mathrm{P} \uplus \mathrm{N}, V_{Q}^{n+1}(a)=\left\{w \sigma \mid w \sigma \in W_{Q}^{n+1}, w \in V(a)\right\}^{29}$

The class of structures Forest $\left(\operatorname{TDEL}_{\mathrm{Q}}\right)$ consists of all models Forest $(M, Q)$ for

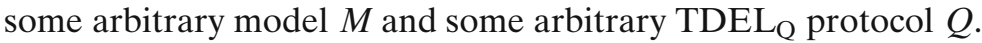

\footnotetext{
${ }^{29} \mathrm{We}$ include here only questions and resolution actions to save space. Other management actions can and should be considered. For instance, here is how clauses for announcement and refinement look:

If $\sigma_{(n+1)}=\varphi$ ! then: (a) $\left(w \sigma, v \sigma^{\prime}\right) \in \approx_{Q}^{n+1}$ iff $\sigma_{(n+1)}=\sigma_{(n+1)}^{\prime}$ and $\left(w \sigma_{n}, v \sigma_{n}^{\prime}\right) \in \approx_{Q}^{n}$; (b) $\left(w \sigma, v \sigma^{\prime}\right) \in$ $\sim_{Q}^{n+1}$ iff $\sigma_{(n+1)}=\sigma_{(n+1)}^{\prime},\left(w \sigma_{n}, v \sigma_{n}^{\prime}\right) \in \sim_{Q}^{n}$, and $\left(w \sigma_{n}, w \sigma_{n}^{\prime}\right) \in{\stackrel{\underline{\underline{\varphi}}}{M_{Q}^{n}}}$;

If $\sigma_{(n+1)}=$ ? then: (a) $\left(w \sigma, v \sigma^{\prime}\right) \in \sim_{Q}^{n+1}$ iff $\sigma_{(n+1)}=\sigma_{(n+1)}^{\prime}$, and $\left(w \sigma_{n}, v \sigma_{n}^{\prime}\right) \in \sim_{Q}^{n}$; (b) $\left(w \sigma, v \sigma^{\prime}\right) \in$ $\approx_{Q}^{n+1}$ iff $\sigma_{(n+1)}=\sigma_{(n+1)}^{\prime},\left(w \sigma_{n}, v \sigma_{n}^{\prime}\right) \in \approx_{Q}^{n}$, and $\left(w \sigma_{n}, v \sigma_{n}^{\prime}\right) \in \sim_{Q}^{n}$;
} 
Using the construction from the previous Definition 19 we define the corresponding temporal structure.

Definition 20 (Generated ETL Model) Let $M=\langle W, \sim, \approx, V\rangle$ be an arbitrary model and let $Q$ be an arbitrary $\mathrm{DEL}_{\mathrm{Q}}$-protocol over $M$ (a set of finite sequences of questioning events closed under initial segments). The Generated ETL Model Forest $(M, Q)=\left\langle\mathrm{H}, \sim, \approx, V^{\prime}\right\rangle$ is defined as follows:

1. $\mathrm{H}=\left\{h \mid\right.$ there is a $w \in W, \sigma \in Q$ with $\left.h=w \sigma \in W_{O}^{\operatorname{len}(\sigma)}\right\}$;

2. For all histories $h, h^{\prime} \in \mathrm{H}$ with $h=w \sigma$ and $h^{\prime}=v \sigma^{\prime}, \quad h \sim h^{\prime} \quad$ iff $\operatorname{len}(\sigma)=\operatorname{len}\left(\sigma^{\prime}\right)$, and $\left(w \sigma, v \sigma^{\prime}\right) \in \sim_{Q}^{\operatorname{len}(\sigma)}$

3. For all histories $h, h^{\prime} \in \mathrm{H}$ with $h \stackrel{Q}{=} w \sigma$ and $h^{\prime}=v \sigma^{\prime}, \quad h \approx h^{\prime} \quad$ iff $\operatorname{len}(\sigma)=\operatorname{len}\left(\sigma^{\prime}\right)$, and $\left(w \sigma, v \sigma^{\prime}\right) \in \approx_{Q}^{\operatorname{len}(\sigma)}$;

4. For each $a \in \mathrm{P} \uplus \mathrm{N}$, and $h=w \sigma \in \mathrm{H}, h \in V_{Q}^{\prime}(a) \quad$ iff $\quad h \in V_{Q}^{\operatorname{len}(\sigma)}(a)$.

Next we give a truth definition for a matching dynamic language, with dynamic actions involving formulas from the static base language only. Here $q$ is used as a variable over all issue management actions.

Definition 21 (Interpretation) Truth of formulas at state $h$ in models Forest $(M, \mathcal{Q}):=\operatorname{Fr}(M, \mathcal{Q})=\langle\mathcal{H}, \sim, \approx, V\rangle$ uses these inductive key clauses:

- $\operatorname{Fr}(M, \mathcal{Q}) \models_{h} K \varphi$ iff $\forall h^{\prime} \in \mathcal{H}: h \sim h^{\prime}$ implies $\operatorname{Fr}(M, \mathcal{Q}) \models_{h^{\prime}} \varphi$;

- $\operatorname{Fr}(M, \mathcal{Q}) \models_{h} Q \varphi$ iff $\forall h^{\prime} \in \mathcal{H}: h \approx h^{\prime}$ implies $\operatorname{Fr}(M, \mathcal{Q})=_{h^{\prime}} \varphi$;

- $\operatorname{Fr}(M, \mathcal{Q}) \models_{h} R \varphi$ iff $\forall h^{\prime} \in \mathcal{H}: h(\sim \cap \approx) h^{\prime}$ implies $\operatorname{Fr}(M, \mathcal{Q}) \models_{h^{\prime}} \varphi$;

- $\operatorname{Fr}(M, \mathcal{Q}) \models_{h} U \varphi$ iff $\forall h^{\prime} \in \mathcal{H}: h=w \sigma, h^{\prime}=w^{\prime} \sigma^{\prime}, w, w^{\prime} \in \operatorname{Dom}(M)$, $\sigma, \sigma^{\prime} \in \mathcal{Q}$, and $\sigma=\sigma^{\prime}$ implies $\operatorname{Fr}(M, \mathcal{Q}) \models_{h^{\prime}} \varphi$;

- $\operatorname{Fr}(M, \mathcal{Q}) \models_{h}\langle q\rangle \varphi$ iff $h q \in \mathcal{H}$ and $\operatorname{Fr}(M, \mathcal{Q}) \models_{h q} \varphi$.

Theorem 7 (Completeness of $\mathbf{T D E L}_{\mathbf{Q}}$ ) The dynamic epistemic question logic $T D E L_{Q}$ of protocol models is completely axiomatizable.

This requires an elaborate, but not essentially changed version of the completeness proof in [17]. The key axioms for $T D E L_{Q}$ are in this setting:

Questions \& Partition:

$$
\langle\varphi ?\rangle Q \psi \leftrightarrow\langle\varphi ?\rangle\rceil \wedge((\varphi \wedge Q(\varphi \rightarrow\langle\varphi ?\rangle \psi)) \vee(\neg \varphi \wedge Q(\neg \varphi \rightarrow\langle\varphi ?\rangle \psi)))
$$

Resolution \& Knowledge:

$$
\langle!\rangle K \varphi \leftrightarrow\langle!\rangle \top \wedge R\langle!\rangle \varphi
$$

The main difference with the earlier reduction axioms is in conjuncts like $\langle\varphi$ ? $\rangle$ T. These now express that the action decribed is available according to the protocol. This procedural information may be sui generis, and not equivalent to any simple precondition formula in the underlying static language. The new logic now encodes epistemic aspects, as for $P A L$ and $D E L$, but also dynamics of issue structure. Cf. [21] for further details. 
We have shown that dynamic logic of questions can easily accommodate global temporal protocols, thus getting closer to a true logic of inquiry. Of course, the main interest is not in our general completeness theorem, but in analyzing concrete protocols, and more detailed logical effects of various restricted question repertoires.

\section{Further Directions}

In this paper, we have shown how dynamic logics of questions can analyze various aspects of private and public inquiry. These systems fit entirely within the methodology of dynamic-epistemic logic, and they seem to form a natural complement to what already exists in this area, making the questions explicit that drive public announcements and other informational events. In line with this first finding, many lines of investigation open up:

Some open problems are close to what we have done here. For instance, we need extensions of our systems to group actions of information and issue management, including common knowledge, and group issue modalities. We have also identified an operation of parallel composition of management actions that needs to be axiomatized. Also, we have raised the open problem of determining the 'schematic validities' behind our dynamic logics, encoding a more perspicuous algebra of issue management. Next, our extension to protocols suggests representation results similar to those known for ' $D E L$ versus ETL' in [29] and [8]. And finally, we need to extend our analysis from Yes/No questions to more complex sorts of questions, such as Whquestions asking for a partition cell in some partition that need not be binary at all.

In addition, we mention a few more general issues on our agenda:

Questioning Games An interesting further development links our dynamic analysis of questions to epistemic games for public announcements by Ågotnes and van Ditmarsch [1]. In such games players have to find optimal announcement in order to reach their, possibly conflicting, epistemic goals. In [1] new solution concepts are proposed for such games, in which the value of a question gets a precise definition. In strategic interactions an optimal question need not be the most informative one, and different preferences may arise in different scenarios. See [2] for a further study of this connection.

Questions, Decisions and Information Another connection, with both information theory and decision theory, defines the 'value of a question' as its role in resolving decision problems by means of the information contained in the available answers. These aspects were introduced and discussed, for instance, in [35-37]. This connection can become even more relevant in a setting that makes explicit use of both an information partition and issue-structure for the answers considered relevant by an agent. 
Further Agent Attitudes: Beliefs and Preferences We have studied the interaction of questions with knowledge. But of course, agents' beliefs are just as important, and we can also merge the preceding analysis with dynamic logics of belief change. In fact, in addition to conveying hard information, asking a question can also be a subtle way of influencing beliefs of agents. For instance, we said earlier that not all questions impart knowledge that the speakers does not know the answer. But we might say that, barring further information, they induce a defeasible belief of the audience that this is the case. Thus, our question dynamics might be added to the $D E L$-style belief logics of van Benthem [26] and Baltag and Smets [6].

Beyond beliefs, questions can also affect other agent attitudes. For instance, a question can give us information about other agents' goals and preferences, and indeed, "Why" questions explicitly concern such reasons for behavior. Just as information dynamics does not stop at purely informational attitudes, but also extends to the way in which agents evaluate situations and actions, the same extension makes sense for questions. This would come out concretely by adding question dynamics to the preference logics of Girard [9] and Liu [20]. ${ }^{30}$

Update, Inference, and Syntactic Awareness Dynamics While DEL has been largely about observation-based semantic information, some recent proposals have extended it to include more finely grained information produced by inference or introspection. The same sort of move makes sense in our current setting. For instance, yet another effect of asking a question is of making agents aware that something is an issue. This does not just make sense in the above epistemic logic-based environment of semantic information. Raising an issue may also just make an agent aware that some proposition is important. In that case, we can think of a finer dynamics of questions, where they increase some current set of 'relevant propositions' whose truth value needs to be determined. This would work well in the syntactic approach to inferential and other fine-grained information in van Benthem and Velazquez-Quesada [33], with questions providing one reason for their acts of 'awareness promotion'. The latter take would also fit well with Hintikka's emphasis on the combination of questions and deductions as driving inquiry. In a dynamic perspective, merging semantic observational information and inferential syntactic information will become even more natural when questions come into play. ${ }^{31}$

\footnotetext{
${ }^{30}$ Indeed, there are formal analogies between our question update operation and the "ceteris paribus' preferences of van Benthem et al. [30].

${ }^{31}$ Consider the realization acts defined in [39] but now take a weaker precondition: instead of requiring that the fact is known we can only ask for the fact to be part of the current issue, or to have been asked. Instead of using the phrase "Observation enables realization" we can consider a weaker slogan "Questioning and observation enables partial realization": [ $p$ ?][ $p \rightarrow q$ !] $\langle+q\rangle I q$.

With these preconditions, a formula can be promoted into the access set even if it was not yet announced. This would also work in the setting that uses both formulas and rules of inference from [38] If some premises of a rule have been announced already and the rest are already part of the current issue, the rule can be applied.
} 
Multi-Agent Behavior over Time We have already indicated that, just as with assertions, questions make most sense in the context of some longer temporal process. A single question is hard to 'place' outside of the setting of some scenario. For instance, questions as much as arguments drive argumentation, and serve as ways of either underpinning assertions, or calling them into doubt. To deal with this formally, we need temporal logics that can talk about sequences of questions and their effects on the current history. Our study of protocols was one step in this direction, but obviously, we also need to make our dynamic logics of questions work in analysis of extended conversation, or especially, games. Another long-term perspective where this makes eminent sense are learning scenarios, where asking successive local questions would seem a very natural addition to the usual input streams of answers (cf. Kelly [19]) to one unchanging grand question which global hypothesis about the actual history is the correct one.

Structured Issues and Agenda Dynamics Surely, both in conversation and in general investigation, the agenda of relevant issues is much more delicate than just some equivalence relation. There are less and more important issues to be solved. Again, this reflects a point on the informational side, where logics of ordered propositions have been proposed to model belief revision and preference ordering. Usually, we are maintaining a 'structured agenda'and it is this agenda that gets modified by successive events of resolving old questions or raising new ones.

There are already models that allow for this sort of dynamics. Girard [9] and Liu [20] use 'priority graphs' of ordered relevant propositions (first proposed and studied in Hajnal et al. [3]) for belief and preference change. Priority graphs can encode a structured family of issues, and they allow for a larger repertoire of inserting or deleting questions. The cited authors have suggested that this would be suited for studying the structured agendas of research programs in the philosophy of science, where theories consist not just of propositions encoding answers to past questions, but also of a representation of those guiding questions. True research skills include asking good questions just as much as giving answers. ${ }^{32}$

\section{Comparisons with Other Approaches}

We have mentioned several other approaches to the logic of questions. There is the tradition of erotetic logic in the sense of Wiśniewski [40], which seems still relevant. Likewise, it would be good to go back to the slightly later classic Belnap and Steel [22] in the light of current dynamic logics.

\footnotetext{
${ }^{32}$ For intermediate level of agenda representation, cf. the neighborhood models of [32].
} 
More directly connected to our approach, we have mentioned the still active program of Hintikka for interrogative logic [15]. Questions are treated here as requests for new information, which function intertwined with deductive indicative moves in 'interrogative tableaux'. There is an extensive theory of answerhood, as well as an analysis of various types of question in a predicatelogical setting, beyond what we have done here. The framework has a number of nice theoretical results, including meta-theorems about the scope of questioning in finding the truth about some given situation. Clearly, several of these results would also be highly relevant to what we are doing here, and a merge of the two approaches might be of interest, bringing out Hintikka's concerns even more explicitly in a dynamic epistemic setting. ${ }^{33}$

Closer to the perspective of this paper, questions have been studied in a more classical dynamic logic setting in [24] and also [34] as PDL programs that effect "focus changes" in the structure of an epistemic model. For more details about this approach and a more extensive comparison, cf. [21].

But the closest comparison to our approach is the current research program of "inquisitive semantics $([7,11]){ }^{34}$ Inquisitive semantics gives propositions an "interrogative meaning" defined in a universe of information states over propositional valuations, with sets of valuations expressing issues. This supports a compositional semantics for the language of propositional logic, where, for instance, a conditional is true in an informational sense if every subset (stronger information state) supporting the antecedent also supports the consequent. Interrogative meanings are then defined in terms of generalized partitions of the set of worlds, where partition cells may now also overlap. This is a significant extension of the traditional issue picture. Based on this semantics, a rich new propositional logic arises that describes valid consequence and other important relations between questions, and for questions and answers. The program has found a variety of applications to natural language semantics and pragmatics.

Inquisitive semantics, too, is based on intuitions of information dynamics, while questions change current partitions, an idea that we have adopted. And indeed, the two systems are close enough for further flow of ideas: for instance, can the dynamic logics of this paper be extended to deal with generalized non-partition issues? ${ }^{35}$ But there is also a major difference. The 'inquisitive logic' matching inquisitive semantics is an intermediate logic with some intuitionistic, rather than classical features. By contrast, our dynamic logics are conservative extensions of classical propositional logic with new dynamic modalities for issue-changing actions. This contrast is not unique to the current setting (cf. [28]). There are more instances of a duality between approaches, one "changing semantics" for some standard language resulting in

\footnotetext{
${ }^{33} \mathrm{~A}$ first study in this comparative line is the Master's thesis [13].

${ }^{34} \mathrm{We}$ cannot do full justice to this fast-growing framework here. For the latest news, see the website: https://sites.google.com/site/inquisitivesemantics/papers-1.

${ }^{35}$ Icard [18] explores a first merged version of the two systems.
} 
a new logic (cf. the intuitionistic approach to information and knowledge [27]), and one "conservatively extending" a classical system with new operators, whose logic is then determined explicitly (cf. epistemic logics for knowledge [25]). In our present setting, the contrast also has to do with the distinction made at the beginning of this paper between "semantics of questions" for natural language and "issue management" in general agency. These are related but not identical concerns, and hence related but not logical systems may be appropriate. Understanding the precise relations between logical systems in these two modes can be delicate. Hence undertaking such a comparison for dynamic logic of questions vis-a-vis inquisitive logic, though clearly very interesting, goes beyond the bounds of this paper.

\section{Conclusion}

The dynamic calculi of questions in this paper show how dynamic-epistemic logic can incorporate a wide range of what we have dubbed 'issue management' beyond mere information handling. Our contribution is showing how this can be done precisely, leading to complete dynamic logics that fit well with existing systems of $D E L$. Moreover, we have indicated how these systems can be used to explore properties of issue management beyond what is found in other logics of questions, including complex epistemic assertions, many agents, explicit dynamics, and temporal protocols. ${ }^{36}$

Even so, we do feel that our systems are only a first step-still far removed from the complex structures of issues that give direction to rational agency. The insight itself that issue management is crucial to the logic of inquiry comes from other traditions, as we have observed, but we hope to have shown that dynamic-epistemic logic has something of interest to contribute.

Acknowledgements We have profited from comments on seminar presentations and previous versions of this paper from Thomas Ågotnes, Alexandru Baltag, Cedric Degremont, Viktoria Denisova, Jan van Eijck, Hans van Ditmarsch, Jeroen Groenendijk, Solomon Feferman, Yacin Hamami, Tomohiro Hoshi, Thomas Icard, Lena Kurzen, Floris Roelofsen, Katsuhiko Sano, Sonja Smets, George Smith, Cristina Unger, and Fernando Velazquez-Quesada.

Open Access This article is distributed under the terms of the Creative Commons Attribution License which permits any use, distribution, and reproduction in any medium, provided the original author(s) and the source are credited.

\section{References}

1. Ågotnes, A., \& van Ditmarsch, H. (2011). What will they say?—Public announcement games. Synthese, 179, 57-85.

\footnotetext{
${ }^{36}$ However, we have not arrived at any definite conclusion about the formal relationships between our dynamic logics and existing alternatives. Perhaps all of them are needed to get the full picture of issue management.
} 
2. Ågotnes, T., van Benthem, J., van Ditmarsch, H., \& Minică, Ş. (2012). Question-answer games. In Proceedings of LOFT-2010. To appear in Journal of Applied Non-Classical Logic.

3. Andréka, H., Ryan, M., \& Schobbens, P. Y. (2002). Operators and laws for combining preference relations. Journal of Logic and Computation, 12(1), 13-53.

4. Baltag, A. (2001). Logics for insecure communication. In J. van Benthem (Ed.), Proceedings of TARK'01 (pp. 111-122).

5. Baltag, A., Moss, L., \& Solecki, S. (1998). The logic of public announcements, common knowledge, and private suspicions. In Proceedings of TARK'98 (p. 43). San Mateo: Morgan Kaufmann.

6. Baltag, A., \& Smets, S. (2007). From conditional probability to the logic of doxastic actions. In Proceedings of TARK '07 (pp. 52-61). New York: ACM.

7. Ciardelli, I., \& Roelofsen, F. (2011). Inquisitive logic. Journal of Philosophical Logic, 40(1), 55-94.

8. Degremont, C. (2009). The temporal mind: Observations on the logic of belief change in interactive systems. Ph.D. thesis, ILLC.

9. Girard, P. (2008). Modal logic for belief and preference change. Ph.D. thesis, Stanford University.

10. Groenendijk, J. (1999). The logic of interrogation: Classical version. In T. Matthews, \& M. Strolovitch (Eds.), SALT IX: Semantics and linguistic theory.

11. Groenendijk, J., \& Roelofsen, F. (2009). Inquisitive semantics and pragmatics. In Proceedings of SPR (Vol. 9). Citeseer.

12. Groenendijk, J., \& Stokhof, M. (1997). Questions. In J. van Benthem, \& A. ter Meulen (Eds.), Handbook of the logic and language. Amsterdam: Elsevier.

13. Hamami, Y. (2010). The Interrogative model of inquiry meets dynamic epistemic logics. Master's thesis, ILLC, University of Amsterdam.

14. Hintikka, J. (2007). Socratic epistemology: Explorations of knowledge-seeking by questioning. Cambridge: Cambridge University Press.

15. Hintikka, J., Halonen, I., \& Mutanen, A. (2002). Interrogative logic as a general theory of reasoning. In D. Gabbay, R. Johnson, H. Ohlbach, \& J. Woods (Eds.), Handbook of the logic of argument and inference. Amsterdam: Elsevier.

16. Holliday, W., Hoshi, T., \& Icard, T. (2011). Schematic validity in dynamic epistemic logic: Decidability. Logic, Rationality, and Interaction, 87-96.

17. Hoshi, T. (2009). Epistemic dynamics and protocol information. Ph.D. thesis, ILLC DS-200908.

18. Icard, T. (2009). Inquisitive semantics and dynamic logic. Manuscript, Stanford Univ.

19. Kelly, K. (1996). The logic of reliable inquiry. New York: Oxford University Press.

20. Liu, F. (2008). Changing for the better-Preference dynamics and agent diversity. Ph.D. thesis, ILLC.

21. Minică, Ş. (2011). Dynamic logic of questions. Ph.D. thesis, ILLC.

22. Belnap, N., \& Steel, T. (1976). The logic of questions and answers. New Haven: Yale University Press.

23. ten Cate, B. (2005). Model theory for extended modal languages. Ph.D. thesis, ILLC.

24. Unger, C., \& Giorgolo, G. (2008). Interrogation in dynamic epistemic logic. In Proceedings of the 13th ESSLLI, Hamburg (pp. 195-202).

25. van Benthem, J. (1993). Reflections on epistemic logic. Logique et Analyse, (34), 5-14.

26. van Benthem, J. (2007). Dynamic logic for belief revision. Journal of Applied Non-Classical Logics, 17(2), 129-155.

27. van Benthem, J. (2009). The information in intuitionistic logic. Synthese, 2(167), 251-270.

28. van Benthem, J. (2011). Logical dynamics of information and interaction. Cambridge: Cambridge University Press.

29. van Benthem, J., Gerbrandy, J., Hoshi, T., \& Pacuit, E. (2009). Merging frameworks for interaction. Journal of Philosophical Logic, 38(5), 491-526.

30. van Benthem, J., Girard, P., \& Roy, O. (2008). Everything else being equal: A modal logic for ceteris paribus preferences. Journal of Philosophical Logic, 38, 83-125.

31. van Benthem, J., \& Liu, F. (2007). Dynamic logic of preference upgrade. Journal of Applied Non-Classical Logic, 17, 157-182.

32. van Benthem, J., \& Pacuit, E. (2011). Dynamic logics of evidence-based beliefs. Studia Logica, $1-32$. 
33. van Benthem, J., \& Velázquez-Quesada, F. R. (2009). Inference, promotion and the dynamics of awareness. ILLC Research Report.

34. van Eijck, J., \& Unger, C. (2010). Computational semantics with functional programming. Cambridge: Cambridge University Press.

35. van Rooij, R. (2003). Questioning to resolve decision problems. Linguistics and Philosophy, 26(6), 727-763.

36. van Rooij, R. (2005). Questions and relevance. In Questions and answers: Theoretical and applied perspectives (Proceedings of 2nd CoLogNET-ElsNET symposium), (ILLC) (pp. 96107).

37. van Rooij, R. (2008). Comparing questions and answers: A bit of logic, a bit of language, and some bits of information. In Formal Theories of Information (pp. 161-192).

38. Velázquez-Quesada, F. (2009). Inference and update. Synthese, 196(2), 283-300.

39. Velázquez-Quesada, F. (2011). Small steps in dynamics of information. Ph.D. Thesis, ILLC.

40. Wiśniewski, A. (1995). The posing of questions. Norwell: Kluwer Academic. 